\title{
Entropy Production in Field Theories without Time-Reversal Symmetry: Quantifying the Non-Equilibrium Character of Active Matter
}

\author{
Cesare Nardini, ${ }^{1,2,4}$ Étienne Fodor, ${ }^{1,3}$ Elsen Tjhung, ${ }^{1}$ Frédéric van Wijland, ${ }^{3}$ Julien Tailleur, ${ }^{3}$ and Michael E. Cates ${ }^{1}$ \\ ${ }^{1}$ DAMTP, Centre for Mathematical Sciences, University of Cambridge, \\ Wilberforce Road, Cambridge CB3 OWA, United Kingdom \\ ${ }^{2}$ SUPA, School of Physics and Astronomy, University of Edinburgh, \\ Peter Guthrie Tait Road, Edinburgh EH9 3FD, United Kingdom \\ ${ }^{3}$ Laboratoire Matière et Systèmes Complexes, UMR 7057 CNRS/P7, Université Paris Diderot, \\ 10 rue Alice Domon et Léonie Duquet, 75205 Paris cedex 13, France \\ ${ }^{4}$ Service de Physique de l'Etat Condensé, CEA, CNRS, Université Paris-Saclay, \\ CEA-Saclay, 91191 Gif-sur-Yvette, France
}

(Received 20 October 2016; revised manuscript received 28 January 2017; published 18 April 2017)

\begin{abstract}
Active-matter systems operate far from equilibrium because of the continuous energy injection at the scale of constituent particles. At larger scales, described by coarse-grained models, the global entropy production rate $\mathcal{S}$ quantifies the probability ratio of forward and reversed dynamics and hence the importance of irreversibility at such scales: It vanishes whenever the coarse-grained dynamics of the active system reduces to that of an effective equilibrium model. We evaluate $\mathcal{S}$ for a class of scalar stochastic field theories describing the coarse-grained density of self-propelled particles without alignment interactions, capturing such key phenomena as motility-induced phase separation. We show how the entropy production can be decomposed locally (in real space) or spectrally (in Fourier space), allowing detailed examination of the spatial structure and correlations that underly departures from equilibrium. For phase-separated systems, the local entropy production is concentrated mainly on interfaces, with a bulk contribution that tends to zero in the weak-noise limit. In homogeneous states, we find a generalized Harada-Sasa relation that directly expresses the entropy production in terms of the wave-vector-dependent deviation from the fluctuation-dissipation relation between response functions and correlators. We discuss extensions to the case where the particle density is coupled to a momentum-conserving solvent and to situations where the particle current, rather than the density, should be chosen as the dynamical field. We expect the new conceptual tools developed here to be broadly useful in the context of active matter, allowing one to distinguish when and where activity plays an essential role in the dynamics.
\end{abstract}

DOI: 10.1103/PhysRevX.7.021007

\section{INTRODUCTION}

Active matter consists of systems where energy is injected at the level of each constituent particle-for instance, to power a self-propelled motion, before being dissipated locally [1]. Interacting assemblies of such particles exhibit a rich phenomenology, ranging from the transition to collective motion [2-6] to the emergence of spatiotemporal chaos [7] and large-scale vortices of selfpropelled composite structures [8,9]. The sustained injection and dissipation of energy at the microscopic level drives the dynamics out of equilibrium. Despite this, it is sometimes difficult to pinpoint a truly nonequilibrium signature in the emergent collective properties: The strong microscopic

Published by the American Physical Society under the terms of the Creative Commons Attribution 4.0 International license. Further distribution of this work must maintain attribution to the author(s) and the published article's title, journal citation, and DOI.
Subject Areas: Soft Matter, Statistical Physics

departure from equilibrium does not necessarily survive in the large-scale physics. This is particularly striking in the emergence of cohesive matter in the absence of cohesive forces through the mechanism of motility-induced phase separation [10-12]. While clearly nonthermal, the largescale dynamics does not lead to steady mass currents, and it closely resembles equilibrium phase separation. Accordingly, many attempts have been made to connect this phenomenology to equilibrium physics [10,13-21].

The departure from equilibrium in active systems has often been studied by introducing effective temperatures as defined by the ratio of response functions to correlators [22]. These reduce to the true temperature in the equilibrium limit because the fluctuation-dissipation theorem then holds [16,23-26]. In particular, effective temperatures have been measured experimentally-for instance, in living systems from the dynamics of injected tracers [27-31]. Generically, however, there is no direct connection between the value of the effective temperature and the nonequilibrium nature of the dynamics encoded in the breakdown 
of time-reversal symmetry (TRS). Another characteristic feature of nonequilibrium systems is the emergence of steady-state currents, whose study has long been of interest [32-37]. On the other hand, TRS breakdown in a steady state is, in general, quantified by the global entropy production rate $\mathcal{S}$ [38]. This can be found, even far from equilibrium, directly from the probability ratio of each realization of the dynamics to its time-reversed counterpart [38-41].

The possibility that temporal or spatial coarse graining of a nonequilibrium system can restore or partially restore TRS, creating an effective equilibrium dynamics, has been theoretically addressed in a number of different studies [42-47]. So far, however, only a few of these address active matter directly [10,48-52]. Our goal in the present work is to understand the connection between emergent phenomena, such as phase separation, and the existence of irreversibility at coarse-grained scales. We address this question by studying the entropy production of stochastic field theories, which describe, at the coarse-grained level, active systems undergoing motility-induced phase separation. Importantly, we progress beyond the evaluation of the global entropy production rate, which, in a steady state, is a single number $\mathcal{S}$, to address the more detailed question of how this is built up of contributions from different regions of real space or reciprocal space. This allows us to develop tools for quantifying deviations from equilibrium in a locally or spectrally resolved fashion, leading to new insight.

To capture the collective physics of self-propelled particles, various continuum theories have been proposed based either on coarse-graining procedures or on symmetry arguments [1]. A prototypical example is "Active Model B" [53], which describes diffusive phase separation between two isotropic phases of active matter at different particle densities. It captures, in stylized form, the coarse-grained manybody dynamics of active particles with either discrete $[10,21]$ or continuous [54,55] angular relaxation of their selfpropulsion direction. (Such particles can phase separate even when their interactions are purely repulsive [12].) Within a gradient expansion of the particle density field $\phi(\mathbf{r})$, at zeroth order, a bulk free-energy density $f(\phi)$ can be constructed, thus providing a mapping to equilibrium [10]. But at the square-gradient level, the mapping to equilibrium is lost and TRS is broken [53,54]. Active Model B thus adds minimal TRS-breaking gradient terms to Model B, which is an equilibrium square-gradient theory widely employed in the theory of critical phenomena [56,57]. This resembles how the KPZ equation [58] was constructed, by extension of the Edwards-Wilkinson model [59], as a prototypical nonequilibrium model of interfacial growth.

The methods used to quantify entropy production rely on path integral representations of stochastic PDEs and weaknoise large deviation theory [60-64]. These have recently been used to address the macroscopic behavior of diffusive systems $[35,65]$. In that context, several stochastic field theories associated via suitable coarse graining [66-69] with lattice [35,63,70] and continuum models [64] have been considered, and large-deviation results for both density $[35,64]$ and current $[32,35,65]$ have been obtained. Other results on stochastic thermodynamics and fluctuation theorems for field theories have been offered [71-74].

In Sec. I, we give a brief review of Active Model B. Then in Sec. III, we define the global entropy production rate $\mathcal{S}$ from the probability of observing a realization of the fluctuating density field $\phi(\mathbf{r}, t)$. A success of $\mathcal{S}$ is to capture in a single number the importance of nonequilibrium effects in a steady state. However, a nonzero value does not allow straightforward physical insight into where or how TRS is broken in the coarse-grained dynamics. To rectify this problem, we introduce a spatial decomposition of the entropy production that allows us to locally resolve the nonequilibrium effects of activity. For a phase-separated state of Active Model B, we find that the main contribution arises from the interfaces. On this basis, one might be tempted to assume that, in homogeneous phases, the nonequilibrium nature of the dynamics becomes unimportant, yielding effective equilibrium behavior $[10,55]$. But in fact this holds only at the mean-field level and is broken by fluctuations, as we show, both directly and by constructing a spectral decomposition of the entropy production. This decomposition generalizes the Harada-Sasa relation (HSR) [47] to active field theories; the HSR relates $\mathcal{S}$ to violations of the fluctuation-dissipation relation. The experimental feasibility to measure entropy production from HSR has already been proven [75-77], while its application to many-body active-matter systems is still elusive. We finally extend our approach to more general systems by considering (in Sec. IV) a diffusive active density field coupled to a momentum-conserving fluid (Active Model $\mathrm{H}$ ) and (in Sec. V) a diffusive model in which the mean current $\mathbf{J}$ has nonzero curl (Active Model B+). In the latter case we can choose whether to calculate $\mathcal{S}$ from $\phi(\mathbf{r}, t)$ as before, or from $\mathbf{J}(\mathbf{r}, t)$ which now contains more information. The results differ, which shows that the entropy production depends on what variables one chooses to retain in a coarse-grained description. In Sec. VI, we give our conclusions.

\section{ACTIVE MODEL B}

We consider a conserved, scalar phase-ordering system at the fluctuating hydrodynamics level. Based on symmetry grounds, the simplest dynamics of its order parameter field $\phi(\mathbf{r}, t)$ satisfies

$$
\dot{\phi}=-\nabla \cdot \mathbf{J}, \quad \mathbf{J}=-\nabla \mu+\mathbf{\Lambda},
$$

where $\mathbf{J}$ is a fluctuating current and $\boldsymbol{\Lambda}$ a spatiotemporal Gaussian white noise field satisfying

$\boldsymbol{\Lambda}=\sqrt{2 D} \Gamma ; \quad\left\langle\Gamma_{\alpha}(\mathbf{r}, t) \Gamma_{\beta}\left(\mathbf{r}^{\prime}, t^{\prime}\right)\right\rangle=\delta_{\alpha \beta} \delta\left(\mathbf{r}-\mathbf{r}^{\prime}\right) \delta\left(t-t^{\prime}\right)$.

Here, the noise strength $D$ is the ratio of the collective diffusivity to the collective mobility; the latter has been set 
to unity in Eq. (1). For systems en route to equilibrium, the deterministic part of the current takes the form

$$
\mathbf{J}_{d} \equiv-\nabla \mu, \quad \mu=\mu_{E} \equiv \frac{\delta \mathcal{F}[\phi]}{\delta \phi} .
$$

This is Model B [56,57]. The chemical potential $\mu_{E}$ derives from a free energy $\mathcal{F}[\phi]$, which is conveniently chosen to be of the $\phi^{4}$ type,

$$
\begin{gathered}
\mathcal{F}[\phi]=\int\left[f(\phi)+\frac{\kappa}{2}|\nabla \phi|^{2}\right] d \mathbf{r}, \\
f(\phi)=\frac{a_{2} \phi^{2}}{2}+\frac{a_{4} \phi^{4}}{4} .
\end{gathered}
$$

Phase separation then arises, at the mean-field level, whenever $a_{2}<0$; here, $a_{4}$ and $\kappa$ are both positive.

For a class of phase-separating active-matter models, it has been argued that the main effect of the activity can be captured at the fluctuating hydrodynamic level by an additional contribution to the chemical potential given, in its simplest form, by

$$
\mu=\mu_{E}+\mu_{A}, \quad \mu_{A} \equiv \lambda|\nabla \phi|^{2} .
$$

Equations (1)-(6) define Active Model B [53,54], which is the simplest coarse-grained description of phase-separating active systems. Note that the explicit coarse graining of self-propelled particles interacting via a density-dependent propulsion speed indeed leads to a closely related dynamics, albeit with more complex density dependence of the various coefficients $[21,54]$.

The defining property of $\mu_{A}$ is that it cannot be written as the functional derivative of any $\mathcal{F}[\phi]$. It represents a nonequilibrium chemical potential contribution, which violates TRS by undermining the free-energy structure of the steady state. Interestingly, while the breakdown of TRS has often been modeled at the microscopic level with nonequilibrium noise terms that directly break fluctuation-dissipation relations, it is the deterministic part of the current $\mathbf{J}$ that deviates from the equilibrium form in Active Model B. This differs from recent studies of the impact of colored noises on Models A and B [78]. Note also that the decomposition of $\mu$ into its equilibrium and nonequilibrium parts is not unique; since the only defining property of $\mu_{A}$ is that it does not derive from a free energy, an arbitrary equilibrium contribution can be moved into it from $\mu_{E}$. This does not change any of the results of the next section, but we will have to be more careful in Sec. IV, where activity affects the stress tensor as well as the chemical potential.

\section{ENTROPY PRODUCTION}

To quantify the breaking of TRS, the main quantity of interest is the noise-averaged, global, steady-state entropy production rate $\mathcal{S}$. According to the precepts of stochastic thermodynamics [38,79], it is defined as [39]

$$
\mathcal{S}=\lim _{\tau \rightarrow \infty} \mathcal{S}^{\tau}, \quad \mathcal{S}^{\tau}=\frac{1}{\tau}\left\langle\ln \frac{\mathcal{P}[\phi]}{\mathcal{P}^{R}[\phi]}\right\rangle,
$$

where $\mathcal{P}$ is the probability of a path $\{\phi(\mathbf{r}, t)\}_{0 \leq t \leq \tau}$, and $\mathcal{P}^{R}$ is the probability of its time-reversed realization. The average $\langle\cdot\rangle$ in Eq. (7) is taken with respect to noise realizations. With suitable ergodicity assumptions, ${ }^{1}$ which we make throughout this paper, averaging over one long trajectory $(\tau \rightarrow \infty)$ with a single noise realization is equivalent to the noise average in Eq. (7). In this case, the angular brackets $\langle\cdot\rangle$ in Eq. (7) can be dropped, and below we do so whenever it suits our purposes. For general initial conditions $\phi(\mathbf{r}, 0)$, this single-trajectory time average will also include a transient contribution that scales sublinearly with its duration $\tau$ and hence does not contribute to the entropy production rate $\mathcal{S}$; the initial condition is then irrelevant. All terms of equilibrium form $\left(\mu_{E}\right)$ contribute only to this transient, as we will see below. Importantly, although individual paths can have negative entropy production, $\mathcal{S}^{\tau}$ as defined in Eq. (7) cannot be negative. Using standard field-theoretical methods $[69,80-82]$, the trajectory weight can be written as

$$
\mathcal{P}[\phi]=\exp [-\mathcal{A}],
$$

where $\mathcal{A}[\phi(\mathbf{r}, t)]$ is the dynamical action.

For Active Model B as studied here, we show below that the global entropy production can be written as

$$
\mathcal{S}=\int\langle\hat{\sigma}(\phi, \nabla \phi, \ldots)\rangle d \mathbf{r},
$$

where $\hat{\sigma}$ is a local function of the field $\phi$ and its derivatives. We then interpret the integrand $\sigma(\mathbf{r}) \equiv\langle\hat{\sigma}\rangle$ as a steadystate local entropy production density. Yet, such an interpretation carries two caveats. First, there are several different possible expressions for $\sigma(\mathbf{r})$ that differ by transient and/or total derivative terms, all giving the same integral $\mathcal{S}$; we return to this issue below. Second, the existence of a local entropy production density seemingly implies additivity of $\mathcal{S}$ over subsystems. However, this is a subtle point which is discussed in Appendix A.

The dynamical action for Active Model B is ${ }^{2}$ [80]

$$
\begin{aligned}
\mathcal{A}[\phi] & =\frac{1}{4 D} \int \mathbb{A}(\mathbf{r}, t) d \mathbf{r} d t, \\
\mathbb{A} & =-\left(\dot{\phi}+\nabla \cdot \mathbf{J}_{d}\right) \nabla^{-2}\left(\dot{\phi}+\nabla \cdot \mathbf{J}_{d}\right) .
\end{aligned}
$$

\footnotetext{
${ }^{1}$ For phase-separated systems, ergodicity is assumed to be subject to specified positions for the interfaces between phases.

${ }^{2}$ An equivalent expression for $\mathcal{A}[\phi]$ is

$$
\mathbb{A}=\left|\nabla^{-1}\left(\dot{\phi}+\nabla \cdot \mathbf{J}_{d}\right)\right|^{2}
$$

with the definition $\nabla^{-1} X \equiv \nabla \nabla^{-2} X$. The latter operation maps a scalar field $X$ to a vector field $\mathbf{Y}$ such that $\nabla . \mathbf{Y}=X$ with a gauge choice $\nabla \wedge \mathbf{Y}=\mathbf{0}$. The two forms for $\mathbb{A}$ in Eqs. (11) and (10) are then related by integration by parts.
} 
Here, the stochastic integral, like all those below, is defined in the Stratonovich sense [83]. In Eq. (11), we have silently omitted a time-symmetric contribution stemming from the Stratonovich time discretization (see Appendixes B and C for details). The integral operator $\nabla^{-2}$ is the functional inverse to the Laplacian (a Coulomb integral). The action $\mathcal{A}$ measures the logarithm of the probability that a solution to Eq. (1) is arbitrarily close to a given path $\{\phi(\mathbf{r}, t)\}_{0 \leq t \leq \tau}$.

We now introduce the time-reversal operation

$$
\begin{aligned}
t & \rightarrow \tau-t, \\
\phi(\mathbf{r}, t) & \rightarrow \phi^{R}(\mathbf{r}, t)=\phi(\mathbf{r}, \tau-t) .
\end{aligned}
$$

This operation defines $\phi^{R}(\mathbf{r}, t)$ as the path found by running a movie of $\phi(\mathbf{r}, t)$ backwards in time. The quantity $\mathcal{P}^{R}$ then represents the probability of observing the trajectory $\phi^{R}(\mathbf{r}, t)$ under the "forward" dynamics (1). Since in these dynamics the deterministic part of the current $\mathbf{J}_{d}$ is a functional of $\phi$, we have $\mathbf{J}_{d}(\mathbf{r}, t) \rightarrow \mathbf{J}_{d}^{R}(\mathbf{r}, t)=\mathbf{J}_{d}(\mathbf{r}, \tau-t)$, as found by substituting Eq. (12) into Eq. (1). Thus, the deterministic current is not reversed, even though the actual current $\mathbf{J}=\mathbf{J}_{d}+\boldsymbol{\Lambda}$ is equal and opposite in the forward and backward paths, because $\dot{\phi}^{R}=-\dot{\phi}$. The forward and backward trajectories are thus likely to require very different realizations of the noise. Put differently, the most probable forward trajectories have small noise contributions so that $\mathbf{J} \simeq \mathbf{J}_{d}$. The total current in the time-reversed trajectory $\mathbf{J}^{R}$, is opposed to the deterministic one $\mathbf{J}^{R} \simeq-\mathbf{J}_{d}^{R}$ so that a very atypical noise realization may be required, such that the probability of observing the reversed trajectories is very small.

Note that the notion of time-reversed trajectories contains a degree of ambiguity. Here, we simply measure the probability of the noise realization required to make the time-reversal of a trajectory $\phi(\mathbf{r}, t)$ a solution of the forward dynamics. This should not be confused with various "conjugacy" operations that instead map the forward dynamics of one system onto the backward dynamics of another [38]. Such mappings typically involve treating some of the parameters in the model as odd under timereversal. This happens in magnetism, where $\mathcal{S}$ can be found by comparing a trajectory in an external magnetic field $\mathbf{B}$ with the time-reversed trajectory in an external magnetic field - B [83]. Such an extension of TRS is therefore mandatory to ensure the absence of entropy production in conservative magnetic systems. In the present case, the external parameters (such as $\lambda$ in Active Model B) must not be chosen to be odd under time-reversal, as this would compare forward trajectories in one system with backward trajectories in one with different dynamics, and indeed different phase equilibria [53]. Therefore, such extensions of TRS are not pertinent to the present paper.

Returning to the main issue, we now observe that the only antisymmetric part in the dynamical action is $\dot{\phi}$. The probability for a forward trajectory to lie arbitrarily close to the time-reversed trajectory $\left\{\phi^{R}(\mathbf{r}, t)\right\}_{0 \leq t \leq \tau}$, as a functional of $\{\phi(\mathbf{r}, t)\}_{0 \leq t \leq \tau}$, is $\mathcal{P}^{R}=\exp \left[-\mathcal{A}^{R}\right]$ with

$$
\begin{aligned}
\mathcal{A}^{R}[\phi] & =\frac{1}{4 D} \int \mathbb{A}^{R}(\mathbf{r}, t) d \mathbf{r} d t, \\
A^{R} & =-\left(\dot{\phi}-\nabla \cdot \mathbf{J}_{d}\right) \nabla^{-2}\left(\dot{\phi}-\nabla \cdot \mathbf{J}_{d}\right),
\end{aligned}
$$

where we have again omitted a time-symmetric contribution arising from the time discretization. Relation (7) for the steady-state entropy production can now be written

$$
\mathcal{S}=\lim _{\tau \rightarrow \infty} \frac{\mathcal{A}^{R}-\mathcal{A}}{\tau}=\lim _{\tau \rightarrow \infty} \frac{1}{4 D \tau} \int\left(\mathbb{A}^{R}-\mathbb{A}\right) d \mathbf{r} d t
$$

Using Eqs. (11) and (13), we get

$$
\mathbb{A}^{R}-\mathbb{A}=4\left(\nabla^{-1} \dot{\phi}\right) \nabla\left(\frac{\delta \mathcal{F}}{\delta \phi}+\mu_{A}\right) .
$$

Performing the spacial integral in Eq. (14) by parts and noting that in the Stratonovich convention $\dot{\phi} \delta \mathcal{F} / \delta \phi=\dot{\mathcal{F}}$, we find that

$$
\begin{aligned}
\mathcal{S} & =-\lim _{\tau \rightarrow \infty} \frac{1}{D \tau} \int \mu(\mathbf{r}, t) \dot{\phi}(\mathbf{r}, t) d \mathbf{r} d t \\
& =-\lim _{\tau \rightarrow \infty} \frac{1}{D \tau}\left[\int \mu_{A}(\mathbf{r}, t) \dot{\phi}(\mathbf{r}, t) d \mathbf{r} d t+\Delta \mathcal{F}\right] .
\end{aligned}
$$

This result is central to all that follows. Here, $\Delta \mathcal{F}=$ $\mathcal{F}(\tau)-\mathcal{F}(0)$ is the difference between the final and initial values of the free-energy functional $\mathcal{F}$. This is, of course, the only term present in the passive limit; the total free energy change then fixes $\tau \mathcal{S}^{\tau}=-\Delta \mathcal{F}$, so that $\mathcal{S}=0$. Note that $\Delta \mathcal{F}$ remains bounded even in active systems because $\mathcal{F}(\tau)$ is bounded below and $\mathcal{F}(0)$ bounded above for sensible initial conditions, in each case by terms linear in the system volume. Hence, this transient contribution always vanishes as $\tau \rightarrow \infty$. Assuming ergodicity, the steady-state entropy production then obeys

$$
\mathcal{S}=-\frac{1}{D} \int\left\langle\mu_{A} \dot{\phi}\right\rangle d \mathbf{r},
$$

where the average $\langle\cdot\rangle$ is now taken over the stationary measure. Importantly (see footnote 1), since we are interested in phase separation and similar situations of broken translational symmetry, we should understand the angle brackets in this expression as being noise averages within an ensemble where interfaces between phases are held stationary in time. We can then define a local steadystate entropy production density

$$
\sigma_{\phi}(\mathbf{r}) \equiv-\frac{1}{D}\left\langle\mu_{A} \dot{\phi}\right\rangle(\mathbf{r}),
$$

whose integral is the entropy production: 

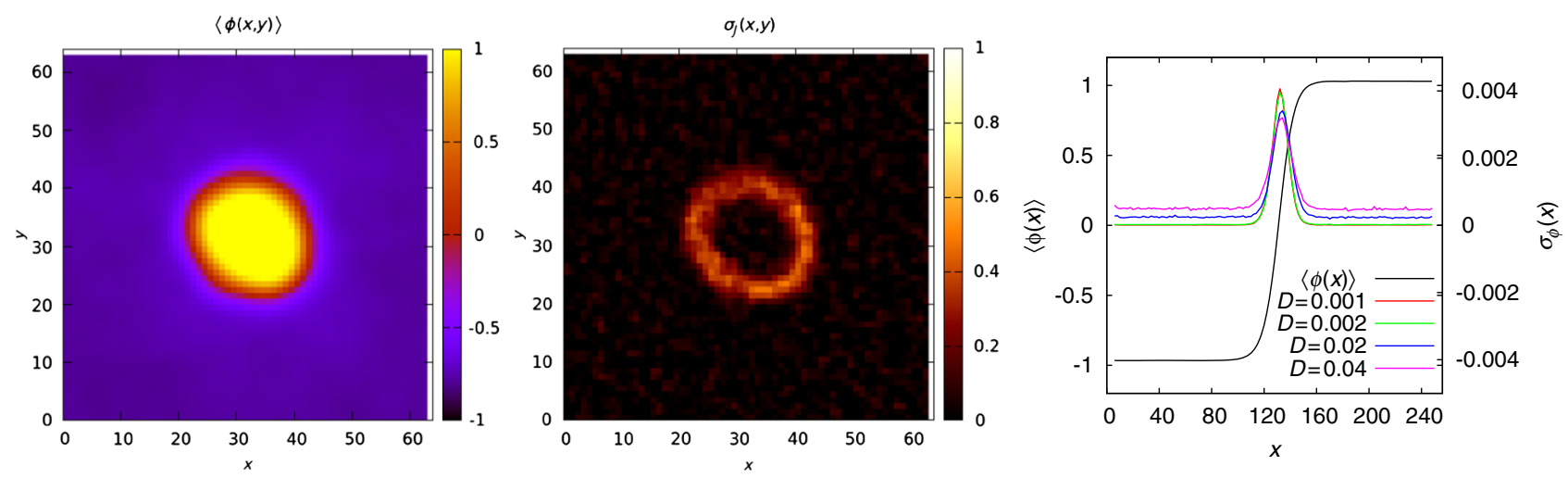

FIG. 1. Left panel: Density map of a fluctuating phase-separated droplet in Active Model B in 2D. Center panel: Local contribution to the entropy production $\sigma(\mathbf{r})$ showing a strong contribution at the interfaces. Right panel: Density and entropy production for a 1D system comprising a single domain wall, for various noise levels $D \ll a_{2}^{2} / 4 a_{4}$. The entropy production is strongly inhomogeneous, attaining a finite value as $D \rightarrow 0$ at the interface between dense and dilute regions and converging to zero in the bulk in this limit. Values of the parameters used are $a_{2}=-0.125, a_{4}=0.125, \kappa=8$, and $\lambda=2$.

$$
\mathcal{S}=\mathcal{S}_{\phi} \equiv \int \sigma_{\phi}(\mathbf{r}) d \mathbf{r}
$$

Here and in Eq. (18), we have added a subscript $\phi$ to distinguish these forms from expressions appearing later (Sec. V) in which the current $\mathbf{J}$ rather than the density field $\phi$ is used to define trajectories.

As already mentioned following Eq. (9) above, there are several other local quantities that have the same integral, $\mathcal{S}$, and hence have equal claim to be called the local entropy production density. Indeed,

$$
-\sigma_{\phi} D \equiv\langle\mu \dot{\phi}\rangle, \quad\left\langle\mu_{A} \dot{\phi}\right\rangle, \quad\left\langle\mathbf{J} . \nabla \mu_{A}\right\rangle, \quad-\left\langle\mathbf{J} . \mathbf{J}_{d}\right\rangle
$$

are all equivalent for the purposes of computing $\mathcal{S}$. The first two forms differ by a transient contribution $\Delta \mathcal{F} / \tau \rightarrow 0$, as do the last two. The latter pair are found from the former by partial integration, differing from them by terms of the form $\nabla . \mathbf{r}_{d, A}$, where $\mathbf{\Upsilon}_{d}=\mathbf{J} \mu$ and $\mathbf{\Upsilon}_{A}=\mathbf{J} \mu_{A}$. Our numerical studies suggest that these alternative candidates for local entropy production are practically indistinguishable in the case of Active Model B. A more complex situation arises for Active Model B+, as described in Sec. V.

\section{A. Spatial decomposition of entropy production}

We now turn to the numerical evaluation of $\sigma_{\phi}(\mathbf{r})$ in Active Model B. We defer to future work any study of the critical region of the model, focusing instead on the weak noise limit (small $D$ ) where sharp interfaces form between high and low density phases, respectively denoted by $\phi_{h}>0$ and $\phi_{l}<0$. Note that $\phi_{h} \neq-\phi_{l}$ unless $\lambda=0$ since activity breaks $\pm \phi$ symmetry in $\mathcal{F}$ [53]. To study phaseseparated states in one dimension, we consider a single domain wall in the center of the system and impose $\nabla \phi=0$ on the distant boundaries. We use finite difference methods with midpoint spatial discretization to integrate Eq. (1) via a fully explicit first-order Euler algorithm. Importantly, the discretized system exactly respects detailed balance whenever $\lambda=0$, as shown in Appendix B where further numerical details are given. For numerical purposes, we evaluate the entropy production density as

$$
\sigma_{\phi}(\mathbf{r})=-\lim _{t \rightarrow \infty} \frac{1}{D t} \int \mu_{A}(\mathbf{r}, t) \nabla^{2} \mu(\mathbf{r}, t) d t
$$

whose equivalence with Eq. (18) is established, within our discretization scheme, in Appendix D.

Our numerics in both one and two dimensions show that the local entropy production density $\sigma_{\phi}(\mathbf{r})$ is strongly inhomogeneous since it is large at the interface between phases but small within these phases (Fig. 1). To quantitatively explain this, we consider the weak noise expansion of the density

$$
\phi=\phi_{0}+\sqrt{D} \phi_{1}+D \phi_{2}+\mathcal{O}\left(D^{3 / 2}\right) .
$$

In the weak noise limit, standard field-theoretical methods, which we outline in Appendix E, show that the dynamics of $\phi_{0}$ and $\phi_{1}$ reduce to

$$
\dot{\phi}_{0}=-\nabla \cdot \mathbf{J}_{d}\left(\phi_{0}\right)
$$

$$
\dot{\phi}_{1}=\nabla^{2}\left[\frac{\delta \mathcal{F}_{0}}{\delta \phi_{1}}+2 \lambda \nabla \phi_{0} \cdot \nabla \phi_{1}\right]+\nabla \cdot \boldsymbol{\Gamma},
$$

where $\boldsymbol{\Gamma}$ is a standard Gaussian white noise as in Eq. (2) and

$$
\mathcal{F}_{0}=\int\left[\left(a_{2}+3 a_{4} \phi_{0}^{2}\right) \frac{\phi_{1}^{2}}{2}+\kappa \frac{\left|\nabla \phi_{1}\right|^{2}}{2}\right] d \mathbf{r} .
$$

This shows, as expected, that the statistics of $\phi_{0,1}$ are independent of $D$ at leading order.

We first consider the case where the mean-field dynamics for $\phi_{0}$ has relaxed to a constant profile. In this case, it follows from Eq. (18) that 


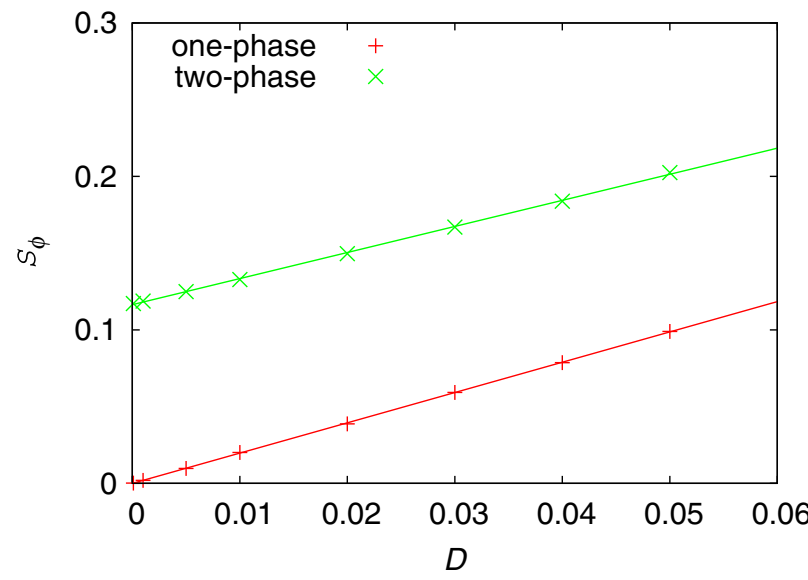

FIG. 2. Total entropy production $\mathcal{S}_{\phi}$ for Active Model B in one dimension for the case where the system consists of a single uniform phase ( $a_{2}>0, a_{4}>0$, red line) and for the case where it shows coexistence between a high-density and a low-density phase $\left(a_{2}<0, a_{4}>0\right.$, green line). [Parameter values are $a_{2}=$ \pm 0.25 for the uniform $(+)$ and phase-separated $(-)$ states, with $a_{4}=0.25, \kappa=4, \lambda=1$.] In the two-phase system, any putative subextensive (interfacial) term scaling as $\sqrt{D}$ would be swamped by the extensive $D^{1}$ contribution from bulk phases.

$$
\sigma_{\phi}(\mathbf{r})=-\lambda \sqrt{D}\left\langle\left|\nabla \phi_{1}\right|^{2} \dot{\phi}_{1}\right\rangle+\mathcal{O}(D) .
$$

Inspection of Eq. (24) shows that the only TRS-breaking term is proportional to $\nabla \phi_{0}$ so that, in a homogeneous state, $\phi_{1}$ has an equilibrium dynamics controlled by the free energy $\mathcal{F}_{0}$. The latter is even in $\phi_{1}$ so that the term of order $\sqrt{D}$ in the expansion (26) of $\sigma_{\phi}$ vanishes by symmetry. We conclude that, in bulk phases, $\sigma_{\phi} \propto D$. We have checked this by simulations in single-phase systems where the total entropy production is indeed shown to scale as $\mathcal{S} \propto D$; see Fig. 2.

In contrast, however, Fig. 2 also shows that the total entropy production $\mathcal{S}$ for a phase-separated system remains finite as $D \rightarrow 0$. This $D^{0}$ term dominates at small enough $D$ and is caused by entropy production localized near the interfaces between phases. This is graphically confirmed in the spatial map of the local entropy production density $\sigma_{\phi}(\mathbf{r})$ shown in Fig. 1, which shows that, as $D \rightarrow 0, \sigma$ vanishes far from interfaces but has a finite contribution in their vicinity. This can be understood from the expansion (22), where $\phi_{0}(\mathbf{r})$ is now a (steady) phase-separated solution of the mean-field dynamics (23), with a prescribed value of $\int \phi(\mathbf{r}) d \mathbf{r}$. Inserting Eq. (22) in the definition (18) of $\sigma_{\phi}$ gives

$$
\begin{aligned}
\sigma_{\phi}(\mathbf{r})= & -\frac{\lambda}{\sqrt{D}}\left|\nabla \phi_{0}\right|^{2}\left\langle\dot{\phi}_{1}\right\rangle-\lambda\left|\nabla \phi_{0}\right|^{2}\left\langle\dot{\phi}_{2}\right\rangle \\
& -2 \lambda \nabla \phi_{0} \cdot\left\langle\nabla \phi_{1} \dot{\phi}_{1}\right\rangle+\mathcal{O}(\sqrt{D}) .
\end{aligned}
$$

In the steady state, $\left\langle\dot{\phi}_{i}\right\rangle=\partial_{t}\left\langle\phi_{i}\right\rangle=0$ so that

$$
\sigma_{\phi}(\mathbf{r})=-2 \lambda \nabla \phi_{0} \cdot\left\langle\dot{\phi}_{1} \nabla \phi_{1}\right\rangle+\mathcal{O}(\sqrt{D})
$$

where the average is taken over the stationary measure of the dynamics (24) for $\phi_{1}$. Given that the statistics of $\phi_{1}$ are, by construction, $D$ independent, it follows that $\sigma_{\phi}(\mathbf{r})=$ $\mathcal{O}\left(D^{0}\right)$ wherever the gradient of the deterministic solution is finite, i.e., at interfaces.

In Fig. 3, we show the dependence of the total entropy production $\mathcal{S}_{\phi}$ on $\lambda$; this scales as $\mathcal{S}_{\phi} \propto \lambda^{2}$. No linear term is possible because it would mean that of the two different Active Model B systems, with other parameters the same but with opposite signs of $\lambda$, one would have negative global entropy production in the steady state. Accordingly, in Eq. (28), the term $\left\langle\dot{\phi}_{1} \nabla \phi_{1}\right\rangle$ must itself be of order $\lambda$, in general. This explains the quadratic scaling but only to leading order in small $\lambda$ (and $D$ ). In practice, we find this scaling over a wide range of $\lambda$ at small $D$ (Fig. 3) and also at larger $D$ (not shown), but we have no explanation for these results at present.

In summary, we have found above that $\sigma_{\phi} \sim D^{0}$, where $\langle\nabla \phi\rangle \neq 0$, whereas $\sigma_{\phi} \sim D$ in bulk phases (Fig. 2). This confirms quantitatively that the TRS breakdown induced by activity plays its largest role at interfaces [21,53]. More generally, the results exemplify how the local entropy production density $\sigma(\mathbf{r})$ can provide a good quantitative tool to understand the breaking of TRS when the system is inhomogeneous. This is likely to be important in situations more complicated than phase separation, including those where activity parameters such as swim speed vary in space (see Ref. [84] and also Sec. V). In such cases, analytical progress could be more difficult, but the numerical evaluation of $\sigma_{\phi}(\mathbf{r})$ should remain tractable. On the other hand, this approach gives little information

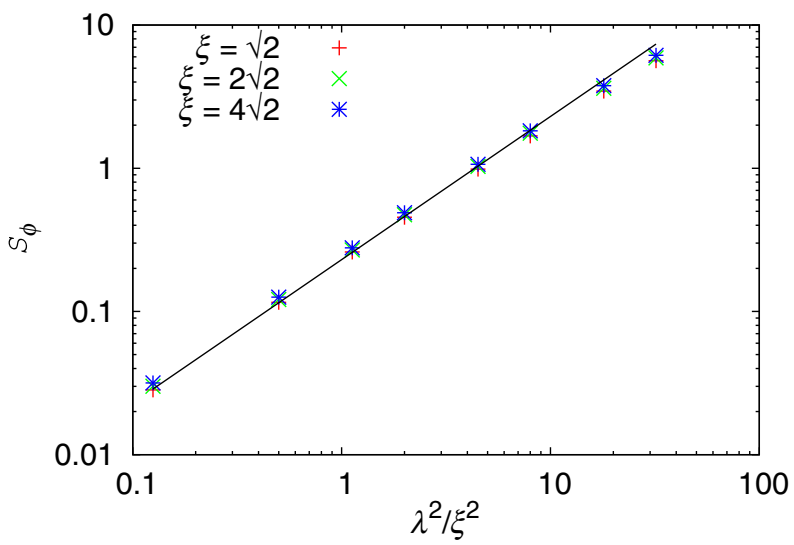

FIG. 3. Scaling of the total entropy production as a function of $\lambda$, showing that $\mathcal{S}_{\phi} \propto \lambda^{2}$, as predicted analytically. Here, $\xi=\sqrt{-\left(\kappa / 2 a_{2}\right)}$ is the passive interface width. Values of the parameters used are $\left(a_{2}=-0.5, a_{4}=0.5, \kappa=2, D=0.001\right)$, $(-0.25,0.25,4,0.001)$, and $(-0.125,0.125,8,0.001)$ for the red, green, and blue points, respectively. 
about the character of TRS breaking in homogeneous phases whose translational invariance forces $\sigma_{\phi}(\mathbf{r})$ to simply be flat. In such cases, spatial structure is captured by correlation functions rather than mean values; we address this next.

\section{B. Spectral decomposition of entropy production from fluctuation and response}

The fluctuation-dissipation theorem (FDT) is a fundamental property of equilibrium dynamics [85]. It states that the response to an external perturbation is entirely determined by spontaneous fluctuations in the absence of the perturbation. For active field theories, and nonequilibrium systems in general, there is no general relation between correlation and response. Thus, violation of the FDT in a coarse-grained description at some scale is proof that activity matters dynamically at this scale since no passive model could ever give rise to such a violation. In equilibrium, the ratio of correlators to response functions is set by the bath temperature. For nonequilibrium dynamics, an effective temperature is sometimes introduced in analogy to the FDT by looking for asymptotic regimes (in time or frequency) where constant values arise for the correlator or response ratio $[25,86]$. One drawback of such a definition of the effective temperature is that it is generically different for every perturbation and observable under scrutiny and therefore bears no universal meaning.

In this section, we provide a quantitative relation between the rate of entropy production $\mathcal{S}$ and the FDT violation for the stochastic field dynamics governed by Eq. (1). We then present detailed numerical results for the case of Active Model B. For an overdamped single particle driven out of equilibrium by an external nongradient force, such a connection was discovered by Harada and Sasa [47]. They showed that the violation of the FDT relating the correlation of the particle position and the response to a constant force provides a direct access to the entropy production rate. Therefore, one important success of the HSR was to identify, among all possible violations of the FDT in a nonequilibrium system, the particular observable and perturbation that quantitatively determine $\mathcal{S}$. The HSR was later generalized to other types of equilibrium dynamics, including the underdamped case and that with temporally correlated noise [87]. HSRs have also been developed for systems with a time-scale separation between fast and slow degrees of freedom [43], and for the density field in a system of particles driven by a time-independent external force field [88]. In active matter, some of us recently announced a generalization of the HSR in a nonequilibrium microscopic model of particles propelled by correlated noises [52].

We consider the correlation function $C$ and the response $R$ to a change of the chemical potential $\mu \rightarrow \mu-h$ :

$$
\begin{aligned}
& C\left(\mathbf{r}_{1}, \mathbf{r}_{2}, t-s\right) \equiv\left\langle\phi\left(\mathbf{r}_{1}, t\right) \phi\left(\mathbf{r}_{2}, s\right)\right\rangle, \\
& \left.R\left(\mathbf{r}_{1}, \mathbf{r}_{2}, t-s\right) \equiv \frac{\delta\left\langle\phi\left(\mathbf{r}_{1}, t\right)\right\rangle}{\delta h\left(\mathbf{r}_{2}, s\right)}\right|_{h=0} .
\end{aligned}
$$

Before outlining the derivation of our generalized HSR, we give the result

$$
\begin{aligned}
\mathcal{S} & =\int \sigma_{\phi}(\mathbf{k}, \omega) d \mathbf{k} d \omega, \\
\sigma_{\phi}(\mathbf{k}, \omega) & \equiv \frac{\omega k^{-2}}{(2 \pi)^{d+1} D}[\omega C(\mathbf{k}, \omega)-2 D R(\mathbf{k}, \omega)],
\end{aligned}
$$

where

$$
\begin{aligned}
& C(\mathbf{k}, \omega) \equiv \int C\left(\mathbf{r}_{1}, \mathbf{r}_{2}, t\right) e^{i \mathbf{k} \cdot\left(\mathbf{r}_{1}-\mathbf{r}_{2}\right)+i \omega t} d \mathbf{r}_{1,2} d t, \\
& R(\mathbf{k}, \omega) \equiv \int R\left(\mathbf{r}_{1}, \mathbf{r}_{2}, t\right) e^{i \mathbf{k} \cdot\left(\mathbf{r}_{1}-\mathbf{r}_{2}\right)} \sin (\omega t) d \mathbf{r}_{1,2} d t .
\end{aligned}
$$

The spectral decomposition of the entropy production rate (30) expresses $\mathcal{S}$ as an integral over Fourier modes of a spectral density $\sigma_{\phi}(\mathbf{k}, \omega)$. This density can formally be seen as the contribution to $\mathcal{S}$ of the modes in $[\mathbf{k}, \mathbf{k}+d \mathbf{k}] \times[\omega, \omega+d \omega]$. It vanishes for thermally equilibrated modes, as enforced by the FDT: $\omega C(\mathbf{k}, \omega)=$ $2 D R(\mathbf{k}, \omega)$. The spectral decomposition is particularly interesting for uniform systems, where the real-space local density $\sigma_{\phi}(\mathbf{r})$ defined in Eq. (18) needs to be constant by translational invariance. It is natural in such systems to instead consider entropy production as a function of wave vector (and perhaps also frequency), and Eq. (30) provides the appropriate tool for doing so. Performing the $\omega$ integral allows one to quantitatively explore whether activity matters dynamically at a given spatial scale. A further asset of the generalized HSR is that it allows one to evaluate $\mathcal{S}$ without complete information about the equations of motion. In particular, as detailed below, we assumed these to lie in the broad class defined by Eq. (1), but we made no assumption about the form of the nonequilibrium chemical potential $\mu_{A}$. This is in contrast to the real-space methods presented in Sec. III A.

We now give the derivation of the HSR result. In terms of the dynamic action $\mathcal{A}^{h}$ of the perturbed dynamics, using the property $\delta \mathcal{P}=-\mathcal{P} \delta \mathcal{A}^{h}$, the response can be written as

$$
R\left(\mathbf{r}_{1}, \mathbf{r}_{2}, t\right)=-\left\langle\left.\phi\left(\mathbf{r}_{1}, t\right) \frac{\delta \mathcal{A}^{h}}{\delta h\left(\mathbf{r}_{2}, 0\right)}\right|_{h=0}\right\rangle .
$$

We need $\mathcal{A}^{h}$ only to first order in $h$ :

$$
\delta \mathcal{A}^{h}=-\frac{1}{2 D} \int h\left(\partial_{t} \phi-\nabla^{2} \mu\right) d \mathbf{r} d t+\mathcal{O}\left(h^{2}\right),
$$

so the spatially diagonal response obeys

$$
R(\mathbf{r}, \mathbf{r}, t)=\frac{1}{2 D}\left\langle\phi(\mathbf{r}, t)\left(\partial_{t} \phi-\nabla^{2} \mu\right)(\mathbf{r}, 0)\right\rangle .
$$



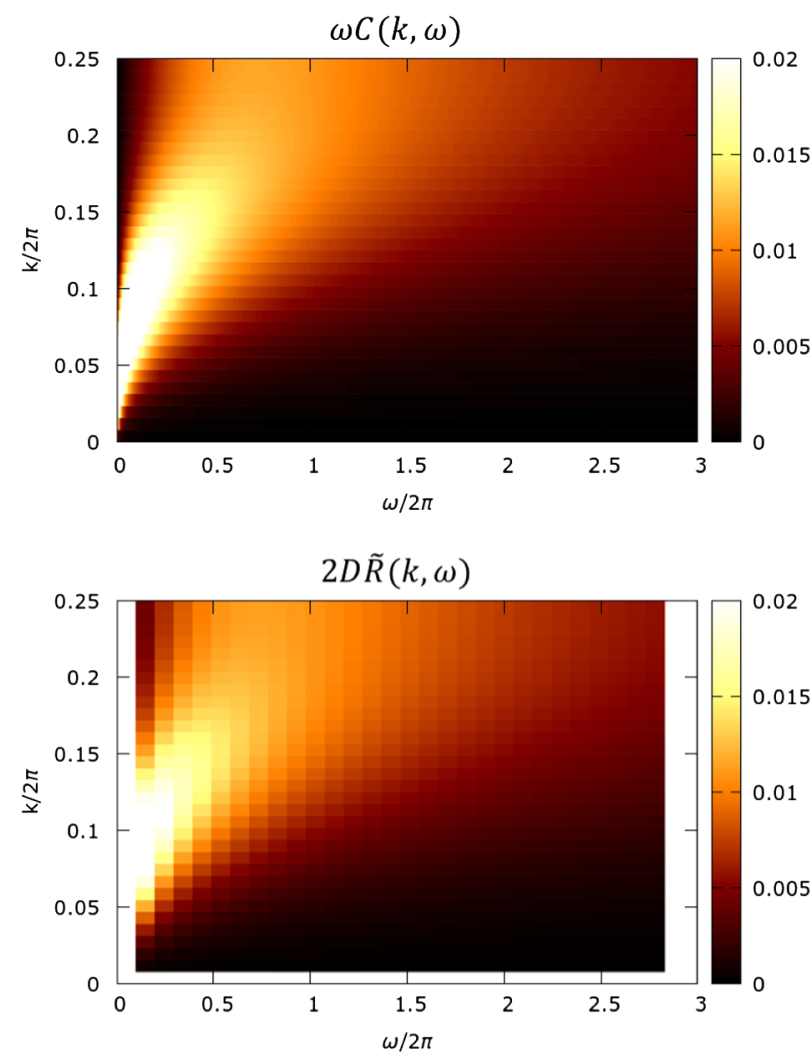

FIG. 4. (a) Correlation $\omega C(\mathbf{k}, \omega)$ and (b) response $2 D R(\mathbf{k}, \omega)$ for Active Model B in one dimension, found by numerical simulations as detailed in the main text. Parameter values are $-a_{2}=a_{4}=0.25, k=4, D=0.05$, and $\lambda=8$.

The time-antisymmetric part follows as

$$
\begin{aligned}
R(\mathbf{r}, \mathbf{r}, t)-R(\mathbf{r}, \mathbf{r},-t) & \\
= & -\frac{1}{D} \partial_{t} C(\mathbf{r}, \mathbf{r}, t) \\
& \quad-\frac{1}{2 D} \nabla_{(2)}^{2}\langle\phi(\mathbf{r}, t) \mu(\mathbf{r}, 0)-\phi(\mathbf{r}, 0) \mu(\mathbf{r}, t)\rangle,
\end{aligned}
$$

where we have used $C(\mathbf{r}, \mathbf{r}, t)=C(\mathbf{r}, \mathbf{r},-t)$ via Eq. (29). Here, $\nabla_{(2)}^{2}$ denotes the Laplacian operator acting on the second spatial variable of the function on which it acts. Comparing with Eq. (17) and ignoring subdominant boundary terms, we deduce

$$
\begin{aligned}
\mathcal{S}= & \frac{1}{D} \lim _{t \rightarrow 0} \int \partial_{t} \nabla_{(2)}^{-2}\{D[R(\mathbf{r}, \mathbf{r}, t)-R(\mathbf{r}, \mathbf{r},-t)] \\
& \left.+\partial_{t} C(\mathbf{r}, \mathbf{r}, t)\right\} d \mathbf{r} d t .
\end{aligned}
$$

This is our generalized Harada-Sasa relation in real space and time. Fourier transforming then yields Eq. (30).

We have numerically computed the spectral decomposition of the entropy production rate for Active Model B, using periodic boundary conditions. We extracted the response using the algorithm of Ref. [89], where two systems, one with unperturbed and the other with perturbed chemical potential, are driven by the same noise realization. This allows a very precise measurement of the response, even without averaging over noise realizations. Nonetheless, a separate simulation for each value of $\mathbf{k}$ and $\omega$ is needed in order to compute the response $R(\mathbf{k}, \omega)$. In contrast, a single run (long enough to ensure good statistical averaging) is sufficient to measure the correlator $C(\mathbf{k}, \omega)$ for all $\mathbf{k}$ and $\omega$ simultaneously. This is why our statistical accuracy is better for $C(\mathbf{k}, \omega)$ than for $R(\mathbf{k}, \omega)$.

In Fig. 4, we plot both quantities in a single-phase system for a wide range of wavelengths and frequencies. Their mismatch spectrally quantifies the breakdown of TRS via the entropy production, as shown in Fig. 5(a). In Fig. 5(b), we present an "effective temperature," defined through $\mathcal{D}(\mathbf{k}, \omega) / D=\omega C(\mathbf{k}, \omega) / 2 D R(\mathbf{k}, \omega)$. This is a popular measure of FDT violations (see above) whose shortcomings are apparent here. In particular, while the effective temperature is peaked at large wave numbers, this is not true of either the FDT mismatch or of $\sigma_{\phi}(\mathbf{k}, \omega)$ itself.
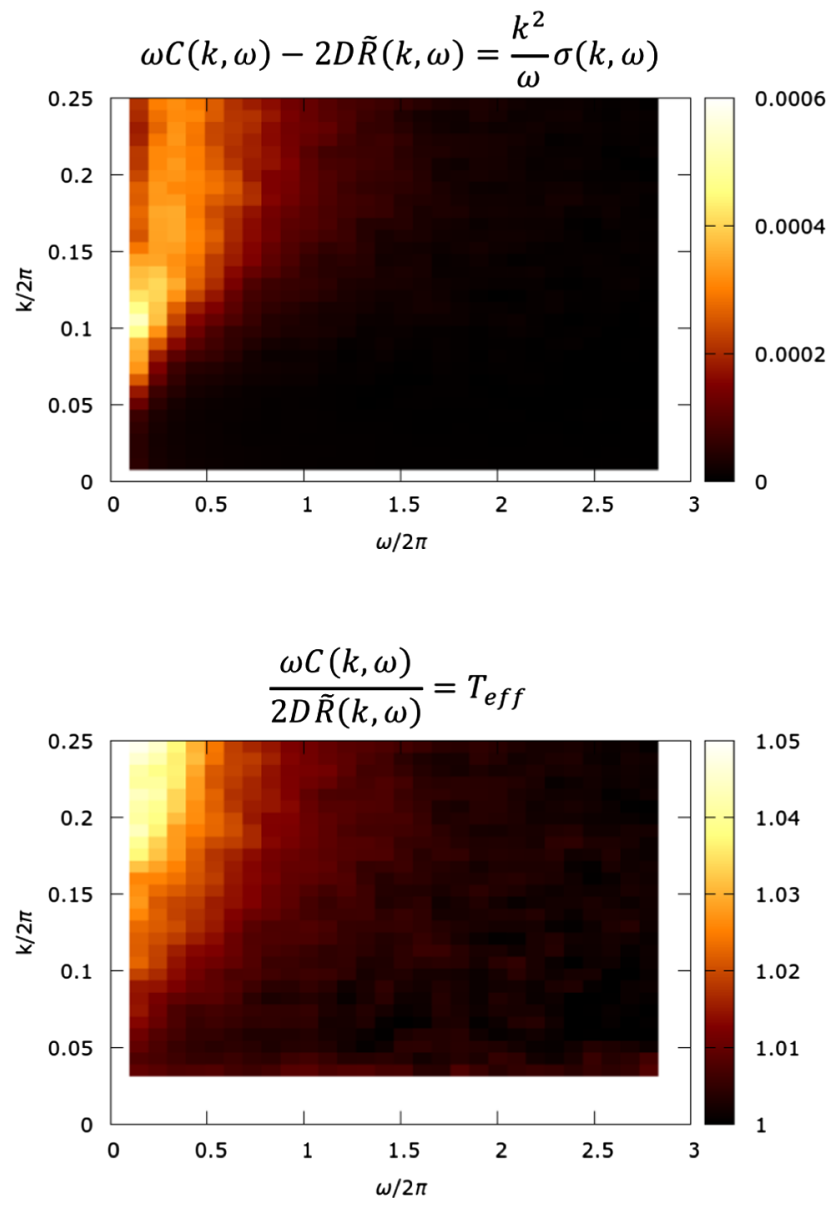

FIG. 5. (a) Correlator-response mismatch $\omega C-2 D \tilde{R} \propto$ $\sigma_{\phi}(\mathbf{k}, \omega) k^{2} / \omega$ and (b) normalized effective temperature $\mathcal{D}(\mathbf{k}, \omega) / D$ as a function of $\mathbf{k}$ and $\omega$ for Active Model B in one dimension. Parameter values as in Fig. 4. (Control simulations for the passive limit $\lambda=0$ recover a statistically insignificant signal for both plots, not shown, confirming that our numerical algorithm respects TRS when present.) 
The additional factor of $\omega / k^{2}$ relating the FDT mismatch to $\sigma_{\phi}(\mathbf{k}, \omega)$ makes the latter highly sensitive to both high-frequency and low-wave-number information. We have checked that the total entropy production $\mathcal{S}=$ $\int \sigma_{\phi}(\mathbf{k}, \omega) d \mathbf{k} d \omega$ nonetheless numerically approaches the same quantity calculated in real space via Eq. (21). The spectral density plot in Fig. 5(a) therefore includes most of the relevant frequency and wave-number range. Deferring fuller investigations to future work, we can conclude that our generalized HSR for Active Model B yields a nontrivial spectral decomposition of the entropy production, including features not captured by the effective temperature paradigm.

Note that measuring the probability of trajectories and their time-reversed counterparts is difficult to do in simulations and even more so in experiments. On the other hand, physicists have an established expertise in measuring FDT both in simulations and experiments. Equation (30) provides a direct link for such measurements to entropy production that awaits exploration for active-matter systems.

\section{ACTIVE MODEL H}

We now turn our attention to Active Model $\mathrm{H}$ in which the diffusive dynamics of $\phi(\mathbf{r}, t)$ is coupled to a momentumconserving fluid with velocity $\mathbf{v}(\mathbf{r}, t)$. This model was first introduced in Ref. [90] to address the role of fluid motion in active phase separation. In that work, it was shown that for so-called contractile activity (where particles pull fluid inward along a polar axis, determined in effect by $\nabla \phi$, and expel it equatorially), domain growth can cease at a length scale where diffusive coarsening is balanced by the active stretching of interfaces. As a generalization to active systems of the well-known Model H [56], this model offers an interesting arena in which to explore coupling of an active scalar to a second field whose dynamics is dissipative, but which would obey TRS without coupling to the active field.

Diffusive dynamics now takes place in the frame of the moving fluid, so Eq. (1) acquires an advective term,

$$
\dot{\phi}+\mathbf{v} \cdot \nabla \phi=-\nabla \cdot \mathbf{J}
$$

where we retain from Active Model B the equations for the diffusive current

$$
\mathbf{J}=\mathbf{J}_{d}+\Lambda, \quad \mathbf{J}_{d}=-\nabla\left(\mu_{E}+\mu_{A}\right) .
$$

We assume the fluid is incompressible and of unit mass density. The thermal Navier-Stokes equation for momentum conservation then reads

$$
\left(\partial_{t}+\mathbf{v} \cdot \nabla\right) \mathbf{v}=\eta \nabla^{2} \mathbf{v}-\nabla p+\nabla \cdot(\boldsymbol{\Sigma}+\tilde{\boldsymbol{\Gamma}}),
$$

with the noise stress specified by [57]

$$
\begin{aligned}
& \left\langle\tilde{\Gamma}_{\alpha \beta}(\mathbf{r}, t) \tilde{\Gamma}_{\gamma \nu}\left(\mathbf{r}^{\prime}, t^{\prime}\right)\right\rangle \\
& \quad=2 \eta D\left(\delta_{\alpha \gamma} \delta_{\beta \nu}+\delta_{\alpha \nu} \delta_{\beta \gamma}\right) \delta\left(\mathbf{r}-\mathbf{r}^{\prime}\right) \delta\left(t-t^{\prime}\right) .
\end{aligned}
$$

(Recall that we set the mobility of the $\phi$ field to unity so that $D=k_{B} T$.) In Eq. (39), the pressure field $p(\mathbf{r}, t)$ subsumes all isotropic stress contributions and enforces fluid incompressibility $(\nabla \cdot \mathbf{v}=0)$. The deviatoric stress tensor $\boldsymbol{\Sigma}$ is traceless and symmetric.

In passive systems, $\boldsymbol{\Sigma}=\boldsymbol{\Sigma}^{E}$ can be derived from a freeenergy functional $\mathcal{F}[\phi]$ by standard procedures [57]; restoring isotropic pressure contributions, one finds

$$
\nabla \cdot \boldsymbol{\Sigma}^{E}=-\phi \nabla \mu_{E} .
$$

For an active system, the relation between the deviatoric stress tensor and the free energy breaks down, taking instead the more general form

$$
\boldsymbol{\Sigma}=\boldsymbol{\Sigma}^{E}+\boldsymbol{\Sigma}^{A},
$$

where $\boldsymbol{\Sigma}^{E}$ obeys Eq. (41). Note that even if activity creates a chemical potential contribution that is of equilibrium form [as arises in motility induced phase separation (MIPS) to zeroth order in gradients], this does not lead to a relation $\nabla \cdot \boldsymbol{\Sigma}^{A}=-\phi \nabla \mu_{A}$ as one might expect from Eq. (41) [90]. This means it is no longer possible, as it was in Active Model B, to shift some arbitrary part of $\mu_{E}$ into $\mu_{A}$ without changing the results. Conversely, one cannot declare all terms in $\mu$ that are of equilibrium form to be part of $\mu_{E}$ as we did for Active Model B in Sec. I; we must now be careful to include in $\mu_{A}$ any chemical potential contribution that does not contribute to the stress via Eq. (41). For this reason, when an external perturbation $\mu \rightarrow \mu-h(\mathbf{r})$ is used to calculate response functions below, we need to specify whether it is $\mu_{A}$ or $\mu_{E}$ that is being perturbed. Note also that applying a perturbation $h(\mathbf{r})$ to the coarse-grained theory need not be equivalent to applying a similar one at the microscopic level; the latter would cause more complicated shifts in both $\mu^{E}$ and $\mu^{A}$ [21].

Active Model H comprises Eqs. (37)-(42). In Ref. [90], it was further assumed that $\mathcal{F}[\phi]$ is given by Eq. (4), from which the deviatoric equilibrium stress $\boldsymbol{\Sigma}^{E}$ takes a dyadic gradient form. This form turns out to be shared by $\boldsymbol{\Sigma}^{A}$ to leading order in gradients:

$$
\boldsymbol{\Sigma}^{E}=-\kappa(\nabla \phi)(\nabla \phi), \quad \boldsymbol{\Sigma}^{A}=-\zeta(\nabla \phi)(\nabla \phi) .
$$

Here, $\zeta$ is a mechanical activity parameter that is positive for extensile and negative for contractile swimmers. Active Model $\mathrm{H}$ thus breaks TRS via two different channels; first through the nonequilibrium chemical potential $\mu_{A}$ in Eq. (38), and second because, for $\zeta \neq 0$, the stress tensor and the free energy are not related by Eq. (41). Our analysis covers the case where Eqs. (4) and (43) hold, as assumed in Ref. [90], but in fact we need no such restrictions on the form of $\mathcal{F}[\phi], \mu_{A}[\phi]$, and $\boldsymbol{\Sigma}^{A}[\phi]$, so long as the latter is a symmetric tensor. 
Using the methods outlined for Active Model B in Sec. I, we can now write the dynamical action density of Active Model $\mathrm{H}$ as

$$
\begin{aligned}
\mathcal{A}= & \frac{1}{4 D} \int\left(\mathbb{A}_{\phi}+\mathbb{A}_{\eta}\right) d \mathbf{r} d t \\
\mathbb{A}_{\phi}= & \left|\nabla^{-1}\left[\left(\partial_{t}+\mathbf{v} \cdot \nabla\right) \phi+\nabla \cdot \mathbf{J}_{d}\right]\right|^{2} \\
\mathbb{A}_{\eta}= & \frac{1}{\eta}\left|\nabla^{-1}\left[\left(\partial_{t}+\mathbf{v} \cdot \nabla\right) v_{\alpha}-\eta \nabla^{2} v_{\alpha}+\partial_{\alpha} p-\partial_{\beta} \boldsymbol{\Sigma}_{\alpha \beta}\right]\right|^{2} \\
& -\frac{1}{2 \eta}\left[\nabla^{-2}\left(\partial_{\alpha} v_{\gamma} \partial_{\gamma} v_{\alpha}-\partial_{\alpha} \partial_{\gamma} \Sigma_{\alpha \gamma}^{A}+\nabla^{2} p\right)\right]^{2}
\end{aligned}
$$

as shown in Appendix $\mathrm{F}$. The pressure $p$ appears in the equations of motion as a Lagrange multiplier for fluid incompressibility; accordingly, $\mathcal{A}$ is as given above for those trajectories $\{\phi(\mathbf{r}, t), \mathbf{v}(\mathbf{r}, t), p(\mathbf{r}, t)\}_{0 \leq t \leq \tau}$ that have $\nabla \cdot \mathbf{v}=0$, but it is infinite for all others. We next construct a time-reversal transformation by supplementing Eq. (12) with

$$
\begin{aligned}
& \rho^{R}(\mathbf{r}, t)=\rho(\mathbf{r}, \tau-t), \\
& v_{\alpha}^{R}(\mathbf{r}, t)=-v_{\alpha}(\mathbf{r}, \tau-t), \\
& p^{R}(\mathbf{r}, t)=p(\mathbf{r}, \tau-t) .
\end{aligned}
$$

We note that both $\mu_{A}$ and $\boldsymbol{\Sigma}^{A}$ must be treated even under time-reversal, in line with the discussion already given in Sec. III. ${ }^{3}$ It is then straightforward to compute the action for the time-reversed dynamics and construct the entropy production via an obvious extension of Eq. (7),

$$
\mathcal{S}_{\phi, \mathbf{v}}=\lim _{\tau \rightarrow \infty} \mathcal{S}^{\tau}, \quad \mathcal{S}^{\tau}=\frac{1}{\tau}\left\langle\ln \frac{\mathcal{P}[\phi, \mathbf{v}]}{\mathcal{P}^{R}[\phi, \mathbf{v}]}\right\rangle,
$$

with the following result:

$$
\mathcal{S}_{\phi, \mathbf{v}}=-\frac{1}{D} \int\left[\mu_{A}\left(\partial_{t}+\mathbf{v} \cdot \nabla\right) \phi+v_{\alpha \beta} \boldsymbol{\Sigma}_{\alpha \beta}^{A}\right] d \mathbf{r} .
$$

Here, $v_{\alpha \beta}=\left(\partial_{\alpha} v_{\beta}+\partial_{\beta} v_{\alpha}\right) / 2$ is the symmetrized velocity gradient tensor. This result reduces to Eq. (18) for Active Model B when $\mathbf{v}=0$, and it applies under the same broad conditions. In particular, it is a steady-state quantity that excludes transient contributions. (The latter would be the only terms present in the phase ordering of passive Model $\mathrm{H}$ without boundary driving [91].)

\footnotetext{
${ }^{3}$ To understand this for Active Model $\mathrm{H}$, consider the stress tensor defined via Eq. (43). At first sight, the above choice of time-reversal appears to contradict the statement in Ref. [90] that $\zeta$ is odd under time-reversal. However, this statement refers to the fact that if one reverses the direction of the fluid flow caused by a swimmer (or set of swimmers) as in Eq. (45), this has the same effect as reversing the sign of $\zeta$, which interchanges the contractile and extensile cases. So, rather than strict time-reversal, this is a conjugacy operation [38] comparing the probabilities of forward and reverse paths in two different systems.
}

The Harada-Sasa relation can be further generalized to the case of Active Model $\mathrm{H}$. To this end, we consider perturbations of the active parts of the chemical potential $\mu_{A} \rightarrow \mu_{A}-h$ and of the stress tensor $\boldsymbol{\Sigma} \rightarrow \boldsymbol{\Sigma}-\varepsilon$, with $\varepsilon$ symmetric and traceless. We introduce the corresponding response functions as

$$
\begin{aligned}
& \mathscr{R}\left(\mathbf{r}_{1}, \mathbf{r}_{2}, t-s\right)=\left.\frac{\delta\left\langle\left(\partial_{t} \phi+v_{\alpha} \partial_{\alpha} \phi\right)\left(\mathbf{r}_{1}, t\right)\right\rangle}{\delta h\left(\mathbf{r}_{2}, s\right)}\right|_{h=0}, \\
& \mathcal{R}\left(\mathbf{r}_{1}, \mathbf{r}_{2}, t-s\right)=\left.\frac{\delta\left\langle v_{\alpha \beta}\left(\mathbf{r}_{1}, t\right)\right\rangle}{\delta \varepsilon_{\alpha \beta}\left(\mathbf{r}_{2}, s\right)}\right|_{\varepsilon=0} .
\end{aligned}
$$

We define the associated autocorrelation functions

$$
\begin{aligned}
& \mathscr{C}\left(\mathbf{r}_{1}, \mathbf{r}_{2}, t\right)=\left\langle\left(\partial_{t} \boldsymbol{\phi}+v_{\alpha} \partial_{\alpha} \phi\right)\left(\mathbf{r}_{1}, t\right)\left(\partial_{t} \boldsymbol{\phi}+v_{\alpha} \partial_{\alpha} \phi\right)\left(\mathbf{r}_{2}, 0\right)\right\rangle, \\
& \mathcal{C}\left(\mathbf{r}_{1}, \mathbf{r}_{2}, t\right)=\left\langle v_{\alpha \beta}\left(\mathbf{r}_{1}, t\right) v_{\alpha \beta}\left(\mathbf{r}_{2}, 0\right)\right\rangle .
\end{aligned}
$$

We demonstrate in Appendix $\mathrm{F}$ that the entropy production rate can be expressed as

$$
\begin{aligned}
\mathcal{S}_{\phi, \mathbf{v}} & =\int\left[\sigma_{\phi}(\mathbf{k}, \omega)+\sigma_{\mathbf{v}}(\mathbf{k}, \omega)\right] d \mathbf{k} d \omega, \\
\sigma_{\phi}(\mathbf{k}, \omega) & =\frac{k^{-2}}{D(2 \pi)^{d+1}}(\mathscr{C}-2 D \mathscr{R})(\mathbf{k}, \omega), \\
\sigma_{\mathbf{v}}(\mathbf{k}, \omega) & =\frac{2 \eta}{D(2 \pi)^{d+1}}(\mathcal{C}-2 D \mathcal{R})(\mathbf{k}, \omega),
\end{aligned}
$$

where

$$
\begin{aligned}
\mathscr{R}(\mathbf{k}, \omega) & \equiv \int \mathscr{R}\left(\mathbf{r}_{1}, \mathbf{r}_{2}, t\right) e^{i \mathbf{k} \cdot\left(\mathbf{r}_{1}-\mathbf{r}_{2}\right)} \cos (\omega t) d \mathbf{r}_{1,2} d t, \\
\mathcal{R}(\mathbf{k}, \omega) & \equiv \int \mathcal{R}\left(\mathbf{r}_{1}, \mathbf{r}_{2}, t\right) e^{i \mathbf{k} \cdot\left(\mathbf{r}_{1}-\mathbf{r}_{2}\right)} \cos (\omega t) d \mathbf{r}_{1,2} d t
\end{aligned}
$$

The coupling of the scalar field with an external velocity field thus requires us to consider two different FDT violations when evaluating $\mathcal{S}$ : one associated with the $\phi$ dynamics and the other with the Navier-Stokes sector. While a pair of such FDTs are satisfied independently in the passive case, the nature of the perturbations and observables entering the HSR could not have been anticipated a priori. In particular, the chemical potential perturbation $\delta \mu=-h(\mathbf{r}, t)$ must act in the diffusive sector alone and not in the thermodynamic stress. This requires $h$ to be considered part of $\mu_{A}$ not of $\mu_{E}$. In consequence, Eq. (50) does not connect with standard expressions for the (transient) entropy production in the passive limit in the way one might have expected. This finding is relevant to the wider agenda of generalizing the HSR to more complicated active field theories, for instance, with orientational order [1]: Since care is required, a relatively formal approach is advisable. Our result demonstrates how the HSR framework singles out, given a set of equations of motion, exactly which FDT violations should be probed to quantify TRS breakdown. 


\section{CIRCULATING CURRENTS}

Active Model B, while abandoning the free-energy structure of its passive counterpart, retains the gradient structure of the deterministic current in Eq. (1): $\mathbf{J}_{d}=-\nabla \mu$. This implies $\nabla \wedge \mathbf{J}_{d} \equiv \mathbf{0}$ so that, at zeroth order in noise, there can be no circulating currents in the steady state. In this section, we outline how our approach generalizes to scalar field theories that allow currents of nonzero curl, as would be needed to capture (for instance) the selfassembled many-body ratched behavior reported for active Brownian particles in Ref. [84].

One such model, which will be addressed in more detail in a separate publication [92], has $\dot{\phi}=-\nabla \cdot\left(\mathbf{J}_{d}+\boldsymbol{\Lambda}\right)$ with

$$
\begin{aligned}
\mathbf{J}_{d} & =-\nabla \tilde{\mu}+\left[\left(\kappa_{0}+\kappa_{1} \phi\right) \nabla^{2} \phi+\nu|\nabla \phi|^{2}\right] \nabla \phi, \\
\tilde{\mu} & =\mu_{B}+\left(\lambda_{0}+\lambda_{1} \phi\right)|\nabla \phi|^{2} \\
\mu_{B} & =-h+a_{2} \phi+a_{3} \phi^{2}+a_{4} \phi^{3}-k \nabla^{2} \phi .
\end{aligned}
$$

This model, which we call Active Model B+, is written here in the presence of a static free-energy contribution $-h(\mathbf{r}) \phi(\mathbf{r})$. In $\mathbf{J}_{d}$, it includes, for completeness, all active terms to order $\left(\phi^{3}, \nabla^{3}\right)$. Of these, only $\kappa_{1}$ and $\nu$ can create nonzero curl. Indeed, only $\nu$ can do this in one dimension, in the sense of creating a nonzero net current around a loop closed via periodic boundary conditions. (This is because $\phi \nabla^{2} \phi$ is reduced to gradient form in one dimension.) By Helmholtz decomposition, $\mathbf{J}_{d}$ can always be written as

$$
\begin{aligned}
\mathbf{J}_{d} & =\mathbf{J}_{d}^{g}+\mathbf{J}_{d}^{r}, \\
\mathbf{J}_{d}^{g} & =-\nabla \mu ; \quad \mathbf{J}_{d}^{r}=\nabla \wedge \mathbf{A},
\end{aligned}
$$

for some choice of scalar field $\mu$ and vector field $\mathbf{A}$, with superscripts $g, r$ standing for gradient and rotational. By similarly decomposing the noise terms, one can also formally define gradient and circulating parts of the total current $\mathbf{J}=\mathbf{J}^{g}+\mathbf{J}^{r}$.

Since $\mathbf{J}^{r}$ is divergence-free, it plays no part in the dynamics of $\phi$. Accordingly, Eq. (7), which defines $\mathcal{S}_{\phi}$, is oblivious to any entropy production arising from circulating currents. To capture this additional source of irreversibility, we now promote $\mathbf{J}(\mathbf{r}, t)$ into an explicit dynamical variable. This leaves $\phi$ dynamically redundant, as it can be reconstructed via $\phi(\mathbf{r}, t)=\phi(\mathbf{r}, 0)-\int_{0}^{t} \nabla \cdot \mathbf{J}(\mathbf{r}, s) d s$. From the point of view of comparing forward and backward trajectories, this corresponds to comparing movies in which the current is recorded (e.g., using tracer particles) rather than just density fluctuations.

If we fix a current trajectory $\{\mathbf{J}(x, t)\}_{0 \leq t \leq \tau}$, the probability that $\mathbf{J}$ is arbitrarily close to it again has an exponential form with action given by

$$
\mathcal{A}_{\mathbf{J}}=\frac{1}{4 D} \int\left|\mathbf{J}-\mathbf{J}_{d}\right|^{2}(\mathbf{r}, t) d \mathbf{r} d t
$$

A straightforward computation shows that the entropy production at the level of currents is

$$
\mathcal{S}_{\mathbf{J}}=\mathcal{S}_{\phi}+\frac{1}{D} \int\left\langle\mathbf{J}^{r} . \mathbf{J}_{d}^{r}\right\rangle(\mathbf{r}) d \mathbf{r} .
$$

For comparison, note that using Eq. (20), $\mathcal{S}_{\phi}$ can be written

$$
\mathcal{S}_{\phi}=\frac{1}{D} \int\left\langle\mathbf{J}^{g} \cdot \mathbf{J}_{d}^{g}\right\rangle(\mathbf{r}) d \mathbf{r} .
$$

There are no cross terms between rotational and gradient contributions because $\int \mathbf{J}^{g} \cdot(\nabla \wedge \mathbf{A}) d \mathbf{r}$ vanishes on partial integration. [This allows either $\mathbf{J}^{r}$ or $\mathbf{J}^{g}$ to be replaced by the full current $\mathbf{J}$ in Eqs. (55) and (56).] The result (56) is compatible with the forms (18) and (21) found above for $\sigma_{\phi}(\mathbf{r})$, although $\mu^{A}$ is now a nonlocal functional ${ }^{4}$ of $\phi$.

We listed in Eq. (20) several, potentially inequivalent, forms for the local entropy production $\sigma_{\phi}$ differing by transient contributions and/or formal entropy currents. Further options arise for the correspondingly defined local quantity $\sigma_{\mathbf{J}}$; we explore these elsewhere [92]. Meanwhile, the simplest case of an additional entropy production arising via Eq, (55) is when the current contains a deterministic rotational part $\mathbf{J}_{d}^{r}$ that is nonvanishing as $D \rightarrow 0$. The resulting contribution to $\mathcal{S}_{\mathbf{J}}$ is

$$
\frac{1}{D} \int\left|\mathbf{J}_{d}^{r}\right|^{2} d \mathbf{r}=\frac{1}{D} \int|\nabla \wedge \mathbf{A}|^{2} d \mathbf{r}
$$

This is a classical form, familiar from the case of external driving, such as a current of charged particles in a circular wire driven by a tangential electromotive force.

Interestingly, the generalized Harada-Sasa relation (30) for $\mathcal{S}_{\phi}$ is insensitive to the additional terms in Eq. (52) and follows from Eq. (18) directly as before, without modification. Indeed, since the circulating currents have no effect on the dynamics of $\phi$, the FDT mismatch of its correlators can give no information about their contribution to the entropy production via the last term in Eq. (55). A connection between Harada-Sasa theory and this term might be made by considering the fluctuations of the curly currents themselves and their response to perturbations that couple to them. We leave this for future investigations.

\section{CONCLUSIONS}

We have studied the violation of TRS in a class of scalar field theories relevant for the description of active matter such as self-propelled particles without alignment interactions. We have presented general tools for addressing the question of whether activity matters dynamicallymeaning, whether it contributes distinctly to TRS-breaking

\footnotetext{
${ }^{4}$ Its calculation involves first constructing $\mu$ as a Coulomb integral of $\nabla \cdot \mathbf{J}$, and then identifying as $\mu_{A}$ the part of it that cannot be represented as a free-energy derivative.
} 
physics, or whether the dynamics at large scales could be reproduced by some (possibly complicated) equilibrium model with TRS. Studying the entropy production allows one to quantify TRS breakdown directly at the coarsegrained level: It unambiguously assesses the extent to which dynamics at this scale is genuinely nonequilibrium.

The main models we considered were Active Models B and $\mathrm{H}[53,90]$. For Active Model B, we defined a local entropy production density and confirmed this to be strongly localized at the interface between phases, with a bulk contribution that is much weaker at low noise levels, by constructing spatial maps to quantify where activity plays a role. We also offered a generalization of the HSR to field theories, quantitatively relating the entropy production rate to the violation of the FDT. This allows entropy production to be spectrally decomposed across spatial and temporal Fourier modes, even in translationally invariant cases where real-space maps convey no useful information.

Assigning wave-number dependence to entropy production allows one to give quantitative underpinning to a statement like "this active system is effectively passive, but only at scales larger than the correlation length." The quantitative link between FDT violation and TRS breakdown reinforces the fact that TRS is, first, a scale-dependent phenomenon and, second, one that depends on which variables are retained in a coarse-grained description. Absence of FDT violation ensures zero entropy production but only if the FDT is tested across the full subset of dynamical fields that we wish to describe. As we showed for Active Model H, the requirements are not always obvious. It might therefore be wise to focus first on the entropy production and, only when its form is clear, turn to HaradaSasa-type constructions to make an explicit connection to FDT violations. This should ensure that no important terms are overlooked. An open task is to construct a HSR capable of detecting the entropy production from circulating currents that are invisible in the dynamics of the density field $\phi(\mathbf{r}, t)$, as exemplified by Active Model B+.

In scalar active matter, the microdynamics is usually very far from equilibrium, despite several approximate mappings to equilibrium. Coarse-grained approximations for the dynamics have been proposed in a number of systems [93-96], at times establishing a surprising connection to equilibrium $[10,97]$. Note the question of whether a given non-TRS dynamics can be approximated, at the coarsegrained level, by one with TRS is a distinct issue from whether the original microscopic dynamics is close to equilibrium [44]. In this article, we provide the tools to decide whether TRS breaking survives coarse graining in active-matter systems.

In some active-matter systems, mesoscopic or macroscopic violations of TRS are obvious-for instance, when fluid vortices are visible at the scale of interest. Our machinery can quantify such violations but is likely to be more useful in cases where they are harder to detect. For example, it would be an interesting followup of this work to apply the theoretical results developed here to Model B coupled to a logistic birth or death term which causes the arrest of phase separation at a particular scale [98]. Particles (representing bacteria) divide in dilute regions and die off in dense ones, manifestly violating TRS at the level of currents. It would be interesting to know if such a model still has finite entropy production when the density field alone is monitored.

Finally, there is clearly a connection between the idea of scale-dependent entropy production and the renormalization-group (RG) concept of "relevance." However, these are not interchangeable since activity could be relevant (changing a universality class) without itself surviving coarse graining (the new class might restore TRS at its fixed point). We hope our work will inform future RG studies both of scalar active field theories and the more complex vector and tensor theories of active matter [1,99-102], for which RG studies are starting to systematically uncover new classes.

\section{ACKNOWLEDGMENTS}

We thank Hugues Chaté, Romain Mari, Frederic MacKintosh, Davide Marenduzzo, Thomas Speck, David Tong, Paolo Visco, and Raphael Wittkowski for illuminating discussions. C. N. acknowledges the hospitality provided by DAMTP, University of Cambridge, while this work was being done. This research has been supported by EPSRC Grant No. EP/J007404. M. E. C. thanks the Royal Society for funding.

\section{APPENDIX A: ADDITIVITY AND LOCALITY OF ENTROPY PRODUCTION}

For Active Model B and its relatives, we established relations such as Eqs. (9) and (17), relating the global steady-state entropy production rate $\mathcal{S}$ to the integral of a local quantity $\sigma(\mathbf{r})$. This shows that the entropy production is additive over regions of space within a larger system. However, if we were to isolate one of these regions as a subsystem, its entropy production rate would depend on what boundary conditions were imposed. Only in the thermodynamic limit, where all subsystems are much larger than the correlation length, do the contributions from such internal boundaries become unimportant.

In this appendix, we consider the case where one specifies fixed information about the time evolution of $\phi$ and its gradients on each internal boundary, which is then shared by the subsystems on either side of that boundary. We examine the additivity of the entropy production in this case and discuss conditions under which it makes sense to view $\sigma(\mathbf{r})$ as a local entropy production density.

We consider a region of space $\Omega_{1}$, with $\Omega_{2}$ its complement; $\partial \Omega$ is the boundary separating the two regions. We restrict our attention to local field theories, defined as those 
in which the trajectories within $\Omega_{1}$ have no further dependence on those in $\Omega_{2}$ once the boundary information $\chi$ is given. The probability for the system to follow a given trajectory $\boldsymbol{\psi}$ that equals $\boldsymbol{\psi}_{1}(\mathbf{r}, t)$ inside $\Omega_{1}$, and $\boldsymbol{\psi}_{2}(\mathbf{r}, t)$ in $\Omega_{2}$, can then be written as

$$
\mathcal{P}[\boldsymbol{\psi}]=\mathcal{P}_{1}\left[\boldsymbol{\psi}_{1} \mid \chi\right] \mathcal{P}_{2}\left[\boldsymbol{\psi}_{2} \mid \chi\right] \mathcal{P}_{\partial \Omega}[\chi],
$$

where $\mathcal{P}_{i}\left[\boldsymbol{\psi}_{i} \mid \chi\right]$ is the conditional probability of finding $\boldsymbol{\psi}_{i}$ in $\Omega_{i}$, given that these fields and their derivatives satisfy some boundary information $\chi(t)$ on $\partial \Omega$, whose unconditional probability is $\mathcal{P}_{\partial \Omega}[\chi]$.

For Active Model B, Eq. (A1) is obviously true if one works with the current $(\boldsymbol{\psi}=\mathbf{J})$ of which the action $\mathcal{A}[\mathbf{J}]$ is a local functional. Less obviously, this is also true working with the density $\phi$, so long as the boundary data $\chi$ contain enough derivatives of $\phi$ both to specify $\left(\dot{\phi}+\nabla \cdot \mathbf{J}_{d}\right)$ on $\partial \Omega$ and to ensure that the Poisson equation $\nabla^{2} f=\left(\dot{\phi}+\nabla \cdot \mathbf{J}_{d}\right)$ has a unique solution inside $\Omega_{i}$. In such cases, one has

$$
\mathcal{P}_{i}\left[\boldsymbol{\psi}_{i} \mid \chi\right]=\frac{W_{i}\left[\boldsymbol{\psi}_{i} \mid \chi\right]}{Z_{i}[\chi]},
$$

where $W_{i}$ is a statistical weight for the conditioned trajectories in $\Omega_{i}$, and $Z_{i}[\chi]$ is its integral over such trajectories. For any local action $\mathcal{A}$, we have $W_{i}=\exp -\mathcal{A}_{i}\left[\boldsymbol{\psi}_{i}\right]$ for trajectories respecting the boundary data and $W_{i}=0$ otherwise; here, $\mathcal{A}_{i}$ is an action integrated only over the domain $\Omega_{i}$. From this, it follows that the statistical weight of the boundary data themselves, $W_{\partial \Omega}[\chi]=Z_{1}[\chi] Z_{2}[\chi]$, and their normalized probability density is

$$
\mathcal{P}_{\partial \Omega}[\chi]=\frac{Z_{1}[\chi] Z_{2}[\chi]}{\int Z_{1}[\chi] Z_{2}[\chi] \mathcal{D}[\chi]}
$$

Substituting Eqs. (A2) and (A3) in Eq. (A1) for the forward probability $\mathcal{P}[\boldsymbol{\psi}]$, and then repeating exactly the same calculation for the backward probability $\mathcal{P}^{R}[\boldsymbol{\psi}]$, gives

$$
\mathcal{S}=\left\langle\mathcal{S}_{1}[\chi]\right\rangle_{\mathcal{P}_{\partial \Omega}}+\left\langle\mathcal{S}_{2}[\chi]\right\rangle_{\mathcal{P}_{\partial \Omega}}+\mathcal{S}_{\chi}
$$

where the entropy production of the boundary process is written

$$
\mathcal{S}_{\chi}=\lim _{\tau \rightarrow \infty} \frac{1}{\tau}\left\langle\ln \frac{\mathcal{P}_{\partial \Omega}}{\mathcal{P}_{\partial \Omega}^{R}}\right\rangle_{\mathcal{P}_{\partial \Omega}} .
$$

In Eq. (A4), the first two terms sum the entropy production in our two subsystems; each of these is first calculated with quenched boundary data $\chi$ as

$$
\mathcal{S}_{i}[\chi]=\lim _{\tau \rightarrow \infty} \mathcal{S}_{i}^{\tau}[\chi] \quad \mathcal{S}_{i}^{\tau}[\chi]=\frac{1}{\tau}\left\langle\ln \frac{\mathcal{P}_{i}}{\mathcal{P}_{i}^{R}}\right\rangle_{\mathcal{P}_{i}\left[\boldsymbol{\psi}_{i} \mid \chi\right]}
$$

and then averaged over $\chi$ [notated as $\langle\cdot\rangle_{\mathcal{P}_{\partial \Omega}}$ in Eq. (A4)].
From Eq. (A2), we obtain

$$
\mathcal{S}_{i}[\chi]=\left\langle\int_{\Omega_{i}} \hat{\sigma}(\boldsymbol{\psi}, \nabla \boldsymbol{\psi}, \ldots) d \mathbf{r}\right\rangle_{\mathcal{P}_{i}\left[\boldsymbol{\psi}_{i} \mid \chi\right]}+\lim _{\tau \rightarrow \infty} \frac{1}{\tau} \ln \frac{Z_{i}^{R}[\chi]}{Z_{i}[\chi]}
$$

with $\hat{\sigma}(\boldsymbol{\psi}, \nabla \boldsymbol{\psi}, \ldots)$ a local function of $\boldsymbol{\psi}$ and its gradients. The first term represents the bulk contribution to $\mathcal{S}$, while the second only depends on the boundary data. This does not vanish, in general, but it does so whenever the boundary information $\chi$ itself exhibits TRS.

Thus, the entropy production rate $\mathcal{S}$ for two systems with a shared boundary is strictly additive only if a movie of events on the boundary itself looks statistically the same when shown in reverse. Generically, this cannot be expected, so there is indeed a finite (subextensive) boundary contribution, which becomes negligible only if the subsystems are large compared to the correlation length.

This type of "qualified additivity" is familiar from the study of systems in equilibrium. Indeed, using arguments that exactly parallel those above, one can show that, in equilibrium, the global entropy defined as

$$
S=-\int \mathcal{P}[\boldsymbol{\psi}] \ln (\mathcal{P}[\boldsymbol{\psi}]) \mathcal{D}[\boldsymbol{\psi}]
$$

obeys [analogous to Eq. (A4)]

$$
S=\left\langle S_{1}[\chi]\right\rangle_{\mathcal{P}_{\partial \Omega}}+\left\langle S_{2}[\chi]\right\rangle_{\mathcal{P}_{\partial \Omega}}+S_{\chi}
$$

with the boundary contribution to the entropy

$$
S_{\chi}=-\int \mathcal{P}_{\partial \Omega}[\chi] \ln \left(\mathcal{P}_{\partial \Omega}[\chi]\right) \mathcal{D}[\chi] .
$$

Moreover, in analogy with Eq. (A7), we find

$$
S_{i}[\chi]=-\left\langle\ln W\left[\boldsymbol{\psi}_{i} \mid \chi\right]\right\rangle_{\mathcal{P}_{i}\left[\boldsymbol{\psi}_{i} \mid \chi\right]}+\ln Z_{i}[\chi] .
$$

In Eqs. (A8)-(A11), $S_{i}, \mathcal{P}$, and $W$ are now functionals of configurations and not trajectories.

These arguments for qualified additivity support the interpretation of $\sigma(\mathbf{r})=\langle\hat{\sigma}\rangle$ in Eq. (9) as a local entropy density, on the basis that $\hat{\sigma}(\boldsymbol{\psi}, \nabla \boldsymbol{\psi}, \ldots)$ stands conceptually in relation to the global entropy production $\mathcal{S}$ just as the equilibrium entropy density $\mathbb{S}(\boldsymbol{\psi}, \nabla \boldsymbol{\psi}, \ldots)$ stands in relation to the global equilibrium entropy $S$. Note that, in the main text, we have mainly focused on the weak noise limit, for which the integral in Eq. (9) is performed at a sufficiently coarse-grained level that long-wavelength fluctuation contributions are unimportant. In equilibrium models, this would correspond to a treatment of $\mathbb{S}(\boldsymbol{\psi}, \nabla \boldsymbol{\psi}, \ldots)$ at the mean-field or density-functional level.

Having established qualified additivity, and given the non-negativity of $\mathcal{S}$, a natural question is whether the entropy production density $\sigma(\mathbf{r})$ is itself non-negative. In partial answer to this, observe first that the various 
contributions in Eq. (A4) are non-negative since the relevant integral fluctuation theorems [38] apply equally to conditional and full probabilities. We thus have

$$
\mathcal{S}_{i}^{\tau}[\chi] \geq 0, \quad \frac{1}{\tau}\left\langle\ln \frac{\mathcal{P}_{\partial \Omega}}{\mathcal{P}_{\partial \Omega}^{R}}\right\rangle_{\mathcal{P}_{\partial \Omega}} \geq 0 .
$$

This might seem to imply the positivity of $\sigma(\boldsymbol{\psi}, \nabla \boldsymbol{\psi}, \ldots)$ when the continuum limit is taken. However, because of the presence of the second term of Eq. (A7), this is actually not a correct inference unless the statistics of the boundary information $\chi$ itself exhibits TRS, so the second term vanishes. This suggests (as is physically reasonable) that any local region of negative $\sigma$ must be driven, through its boundary, by a larger, positive entropy production happening elsewhere. We defer to future work the question of whether such situations can arise in practice for the active field theories studied here.

\section{APPENDIX B: DISCRETIZED DYNAMICS}

In this appendix, we detail how to perform spatial discretization such that detailed balance is always recovered in the equilibrium limit for Active Model B. This is the discretization we used to numerically integrate the model, giving the results in Sec. I.

We consider here a 1D Model B $\left(\mu_{A}=0\right)$, although it is easy to extend the results of this appendix to higher dimensions. We consider a system of finite width $L$ such that $x \in[0, L]$ with periodic boundary conditions. We discretize $x$ into $N$ lattice points with equal lattice spacing $\Delta$ so that $N \Delta=L$, and the density field as $\phi(x, t) \rightarrow \phi_{i}(t)$, where $i=1,2, \ldots, N ; \phi_{i}$ is the value of $\phi$ at $x=i \Delta$. The dynamics then becomes

$$
\partial_{t} \phi_{i}=\nabla^{2} \frac{\partial \mathcal{F}}{\partial \phi_{i}}+\sqrt{2 D} \nabla \eta_{i},
$$

with $\left\langle\eta_{i}(t) \eta_{j}\left(t^{\prime}\right)\right\rangle=\delta_{i j} \delta\left(t-t^{\prime}\right)$. We now choose the following discretization for the gradient operator $\nabla$ :

$$
\nabla \psi_{i}=\frac{\psi_{i+1}-\psi_{i-1}}{2 \Delta},
$$

for any discrete fields $\psi_{i}$, which implies the discretization for the Laplacian operator:

$$
\nabla^{2} \psi_{i}=\frac{\psi_{i+2}-2 \psi_{i}+\psi_{i-2}}{4 \Delta^{2}} .
$$

To show that the detailed balance is satisfied, we substitute Eqs. (B2) and (B3) into Eq. (B1) to obtain

$$
\partial_{t} \phi_{i}=-\frac{1}{4 \Delta^{2}} A_{i j} \frac{\partial \mathcal{F}}{\partial \phi_{j}}+\frac{\sqrt{2 D}}{2 \Delta} B_{i j} \eta_{j},
$$

where the matrices $A$ and $B$ are given by

$$
A=\left(\begin{array}{cccccc}
2 & 0 & -1 & & & \\
0 & 2 & 0 & -1 & & \\
-1 & 0 & 2 & 0 & -1 & \\
& & & \ddots & & \\
& & & & \ddots & \\
& & & -1 & 0 & 2
\end{array}\right)
$$

and

$$
B=\left(\begin{array}{ccccc}
0 & 1 & & & \\
-1 & 0 & 1 & & \\
& -1 & 0 & -1 & \\
& & & \ddots & \\
& & & -1 & 0
\end{array}\right),
$$

with zero elements where not explicitly written. We observe $B B^{T}=B^{T} B=A$, which is the condition for this discretized dynamics to obey exactly detailed balance $[83,103]$. Using the same discretization for the active term $\mu_{A}$, this ensures that TRS is respected fully in the limit $\mu_{A} \rightarrow 0$.

\section{APPENDIX C: TIME-SYMMETRIC CONTRIBUTION TO $\mathcal{A}$}

The time-symmetric contribution that was omitted from Eq. (11) depends on the prescription used for defining stochastic integrals (Ito, Stratonovich, or intermediate [104-107]) and also on the spatial discretization used; indeed, the subscript $S$ denotes our adopted Stratonovich convention. As discussed in Appendix B above, we use midpoint discretization to maintain exact TRS in the passive limit. Therefore, here we present only the form of this term, notated $\mathcal{A}_{S}$ in the following, for Active Model $B$ in the Stratonovich convention with midpoint discretization and the choice $\mu_{A}=\lambda|\nabla \phi|^{2}$. This is straightforwardly obtained from Eq. (5.15) in Ref. [104] as

$$
\mathcal{A}_{S}=\mathcal{A}_{S}^{R}=-\frac{1}{2 \Delta^{2}} \sum_{i}\left[f^{\prime \prime}\left(\phi_{i}\right)+\frac{3 \kappa}{4 \Delta^{2}}\right] .
$$

This is divergent as $\Delta \rightarrow 0$, both in the part that depends on $f^{\prime \prime}\left(\phi_{i}\right)$ and in the second, constant contribution. However, one still has $\mathcal{A}_{S}-\mathcal{A}_{S}^{R}=0$, as promised, which is all we need for the results of the main text. The use of any other convention and/or discretization would give the same final result for $\mathcal{S}$ once the counterpart of $\mathcal{A}_{S}-\mathcal{A}_{S}^{R}$, which is no longer zero, is properly worked out. 


\section{APPENDIX D: SPATIAL DISCRETIZATION}

The result (21) follows from Eq. (18) only if $\left\langle\mu_{A} \nabla . \boldsymbol{\Lambda}\right\rangle=0$. This would hold automatically if the stochastic integrals were interpreted in the Ito convention in which the time derivative is evaluated at the start of the time step; however, in this paper, we use the (mid-time-step) Stratonovich convention, which anticipates part of the subsequent increment so that $\dot{\phi}$ cannot be simply replaced by $\nabla^{2} \mu$ to give Eq. (21) from Eq. (18). Here, we only address the special case of Active Model $\mathrm{B}$ in one dimension. We show that Eq. (21) still holds, so long as we employ midpoint spatial discretization.

Any stochastic integral in the Stratonovich convention can be transformed into one in the Ito convention. However, subtleties arise when dealing with stochastic PDEs; these are closely linked to the $\mathcal{A}_{S}$ term in the dynamical action discussed in Appendix $\mathrm{C}$ above. Let us first consider a stochastic differential equation in the form $\left[x_{i} \in \mathbb{R}, x=\left(x_{1}, \ldots, x_{n}\right)\right]$

$$
\dot{x}_{i}=a_{i}(x)+b_{i j} \eta_{j},
$$

where $\left\langle\eta_{i}(t) \eta_{j}(s)\right\rangle=\delta_{i j} \delta(t-s)$. We want to consider the following Stratonovich integral:

$$
\mathcal{I}_{i l}=\int_{0}^{t} f_{i}(x(s)) \eta_{l}(s) d s,
$$

where $x(s)$ satisfies Eq. (D1). Here, $\mathcal{I}_{i l}$ can be converted into an Ito plus a nonstochastic (Riemann) integral as follows [108]:

$$
\mathcal{I}_{i l}=\int_{0}^{t} f_{i}(x(s)) \cdot \eta_{l}(s) d s+\mathcal{I}_{i l}^{\text {conv }},
$$

where denotes that the integral has to be understood in the Ito sense, and

$$
\mathcal{I}_{i l}^{\text {conv }}=\frac{1}{2} \int_{0}^{t} \frac{\partial f_{i}}{\partial x_{j}}(x(s)) b_{j l} d s .
$$

This result, when formally generalized to the case of Active Model B, produces ill-defined formulas, involving the square of a Dirac delta. In order to progress, we then midpoint-discretize the dynamics (1)-(4) as in Appendix B, with periodic boundary conditions. We have

$$
\dot{\phi}_{i}=\frac{\mu_{i+2}-2 \mu_{i}+\mu_{i-2}}{4 \Delta^{2}}+\frac{\sqrt{2 D}}{2 \Delta} B_{i j} \eta_{j},
$$

where $B$ obeys Eq. (B6), that is, $B_{i j}=\delta_{i+1, j}-\delta_{i-1, j}$. Now consider the part of entropy production coming from the stochastic integral:

$$
\mathcal{I}=-\frac{\Delta}{2 t D \Delta} \sum_{i} \int_{0}^{t} \mu_{A, i}\left(\eta_{i+1}-\eta_{i-1}\right) .
$$

Applying Eq. (D4) and using the discrete form of $\mu_{A}$,

$$
\mu_{A, i}=\lambda\left(\frac{\phi_{i+1}-\phi_{i-1}}{2 \Delta}\right)^{2},
$$

we have $\mathcal{I}^{\text {conv }}=0$. We conclude that, with midpoint spatial discretization, the expression for the entropy production (18) can be equally interpreted in Ito or Stratonovich conventions. Then, using the nonanticipating property of the Ito convention, Eq. (18) implies Eq. (21).

This result requires the integrand $\mu_{A, i}$ in Eq. (18) to depend only on $i \pm 1$ and not on $i \pm 2$. If we wanted to write the entropy production in the equivalent form

$$
\mathcal{S}=-\frac{1}{D} \int\langle\mu \dot{\phi}\rangle(\mathbf{r}) d \mathbf{r},
$$

we would find (with midpoint spatial discretization) that the conversion from Stratonovich to Ito brings in a term

$$
-\lim _{t \rightarrow \infty} \frac{1}{t} \frac{\Delta}{2 \Delta^{2}} \sum_{i} \int\left[\frac{3 \kappa}{4 \Delta^{2}}+f^{\prime \prime}\left(\phi_{i}\right)\right] d t,
$$

which does not admit a continuous limit for $\Delta \rightarrow 0$. However, this divergence cancels against another term,

$$
-\frac{1}{D} \int\langle\mu \cdot \nabla \Lambda\rangle(\mathbf{r}) d \mathbf{r},
$$

which appears in the Ito but not the Stratonovich integral for $\mathcal{S}$. These facts have been checked in our numerical simulations of Active Model B. Let us finally observe that Eq. (D9) is actually proportional to $\mathcal{A}_{S}$; see Eq. (C1). However, this is nongeneric and due to the fact that the noise in the dynamics is additive and $B B^{T}=B^{T} B=A$.

We conclude by noting that analogous apparent divergences would be found by using other types of spatial discretization or Fourier truncations in the numerical computation of the entropy production.

\section{APPENDIX E: SMALL NOISE EXPANSION}

We expand the dynamic action with the $D$ expansion of $\phi$ in Eq. (22). Since we use this action only for bookkeeping, we choose the simpler Ito convention in this appendix. We have

$$
\begin{aligned}
\mathcal{A}[\phi]= & -\frac{1}{4 D} \int\left[\dot{\phi}_{0}+\nabla \cdot \mathbf{J}_{d}\left[\phi_{0}\right]\right] \nabla^{-2}\left[\dot{\phi}_{0}+\nabla \cdot \mathbf{J}_{d}\left[\phi_{0}\right]\right] d \mathbf{r} d t \\
& -\frac{1}{2 \sqrt{D}} \int\left[\dot{\phi}_{0}+\nabla \cdot \mathbf{J}_{d}\left[\phi_{0}\right]\right] \nabla^{-2} \\
& \times\left[\dot{\phi}_{1}-\nabla^{2}\left(\frac{\delta \mathcal{F}_{0}}{\delta \phi_{1}}+2 \lambda \nabla \phi_{0} \cdot \nabla \phi_{1}\right)\right] d \mathbf{r} d t \\
& -\frac{1}{4} \int\left[\dot{\phi}_{1}-\nabla^{2}\left(\frac{\delta \mathcal{F}_{0}}{\delta \phi_{1}}+2 \lambda \nabla \phi_{0} \cdot \nabla \phi_{1}\right)\right] \nabla^{-2} \\
& \times\left[\dot{\phi}_{1}-\nabla^{2}\left(\frac{\delta \mathcal{F}_{0}}{\delta \phi_{1}}+2 \lambda \nabla \phi_{0} \cdot \nabla \phi_{1}\right)\right] d \mathbf{r} d t+\mathcal{O}(\sqrt{D}),
\end{aligned}
$$


where we have used the definition of $\mathcal{F}_{0}$ in Eq. (25). In the small noise limit, the first term in Eq. (E1) must vanish to avoid any divergence, yielding the mean-field equation $\dot{\phi}_{0}=-\nabla \cdot \mathbf{J}_{d}\left(\phi_{0}\right)$. The second term thus also vanishes, and the third one corresponds to the dynamics of $\phi_{1}$ given by Eq. (24).

\section{APPENDIX F: HARADA-SASA FOR ACTIVE MODEL $\mathrm{H}$}

We first prove that the action of Active Model $\mathrm{H}$ is given by Eq. (44). Introducing the noise vector $r_{\alpha}=\partial_{\beta} \Gamma_{\alpha \beta}$, its correlations read

$$
\begin{aligned}
& \left\langle\Upsilon_{\alpha}(\mathbf{r}, t) \Upsilon_{\beta}\left(\mathbf{r}^{\prime}, t^{\prime}\right)\right\rangle \\
& \quad=-2 \eta D\left(\delta_{\alpha \beta} \nabla^{2}+\partial_{\alpha} \partial_{\beta}\right) \delta\left(\mathbf{r}-\mathbf{r}^{\prime}\right) \delta\left(t-t^{\prime}\right) .
\end{aligned}
$$

The dynamic action corresponding to the fluid dynamics is $\mathcal{A}_{\eta}=\frac{1}{2} \int_{\mathbf{r}, \mathbf{r}^{\prime}, t, t^{\prime}} \mathbf{r}_{\alpha}(\mathbf{r}, t) \Xi_{\alpha \beta}\left(\mathbf{r}-\mathbf{r}^{\prime}, t-t^{\prime}\right) \mathbf{\Upsilon}_{\beta}\left(\mathbf{r}^{\prime}, t^{\prime}\right)$,

where

$$
\begin{aligned}
& \int_{\mathbf{r}^{\prime \prime}, t^{\prime \prime}} \Xi_{\alpha \gamma}\left(\mathbf{r}-\mathbf{r}^{\prime \prime}, t-t^{\prime \prime}\right)\left\langle\Upsilon_{\gamma}\left(\mathbf{r}^{\prime \prime}, t^{\prime \prime}\right) \mathbf{\Upsilon}_{\beta}\left(\mathbf{r}^{\prime}, t^{\prime}\right)\right\rangle \\
& \quad=\delta_{\alpha \beta} \delta\left(\mathbf{r}-\mathbf{r}^{\prime}\right) \delta\left(t-t^{\prime}\right) .
\end{aligned}
$$

We get the explicit expression for $\Xi$ as

$$
\begin{aligned}
& \Xi_{\alpha \beta}\left(\mathbf{r}-\mathbf{r}^{\prime}, t-t^{\prime}\right) \\
& \quad=-\frac{1}{2 \eta D} \nabla^{-2}\left(\delta_{\alpha \beta}-\frac{1}{2} \nabla^{-2} \partial_{\alpha} \partial_{\beta}\right) \delta\left(\mathbf{r}-\mathbf{r}^{\prime}\right) \delta\left(t-t^{\prime}\right) .
\end{aligned}
$$

Using incompressibility, the contribution of the fluid dynamics to the total action follows as in Eq. (44).

We now consider the derivation of the generalized Harada-Sasa relation for Active Model $\mathrm{H}$, stated in Eq. (50). It is useful to start from an expression of the entropy production equivalent to Eq. (47) up to boundary terms:

$$
\mathcal{S}_{\phi, \mathbf{v}}=-\frac{1}{D} \int\left\langle\mu\left(\partial_{t}+\mathbf{v} \cdot \nabla\right) \phi+v_{\alpha \beta} \boldsymbol{\Sigma}_{\alpha \beta}\right\rangle d \mathbf{r} .
$$

This can be rewritten as

$$
\begin{aligned}
\mathcal{S}_{\phi, \mathbf{v}}= & -\frac{1}{2 D} \lim _{t \rightarrow 0} \int\left\langle\mu(\mathbf{r}, 0)\left[\left(\partial_{t}+v_{\alpha} \partial_{\alpha}\right) \phi\right](\mathbf{r}, t)\right. \\
& +\mu(\mathbf{r}, t)\left[\left(\partial_{t}+v_{\alpha} \partial_{\alpha}\right) \phi\right](\mathbf{r}, 0) \\
& \left.+v_{\alpha \beta}(\mathbf{r}, 0) \boldsymbol{\Sigma}_{\alpha \beta}(\mathbf{r}, t)+v_{\alpha \beta}(\mathbf{r}, t) \boldsymbol{\Sigma}_{\alpha \beta}(\mathbf{r}, 0)\right\rangle d \mathbf{r} .
\end{aligned}
$$

The dynamics perturbed as $\mu_{A} \rightarrow \mu_{A}-h$ is given by

$$
\left(\partial_{t}+v_{\beta} \partial_{\beta}\right) \phi=-\partial_{\alpha} J_{\alpha}, \quad J_{\alpha}=-\partial_{\alpha}(\mu-h)+\Gamma_{\alpha} .
$$

The corresponding dynamical action shift at linear order in $h$ reads

$\delta \mathcal{A}=-\frac{1}{2 D} \int h\left[\left(\partial_{t}+v_{\beta} \partial_{\beta}\right) \phi-\partial_{\alpha \alpha}^{2} \mu\right] d \mathbf{r} d t+\mathcal{O}\left(h^{2}\right)$.

From this shift follows the response

$$
\begin{aligned}
\mathscr{R}(\mathbf{r}, \mathbf{r}, t)= & \frac{1}{2 D}\left\langle[ ( \partial _ { t } + v _ { \alpha } \partial _ { \alpha } ) \phi ] ( \mathbf { r } , t ) \left[\left(\partial_{t}+v_{\beta} \partial_{\beta}\right) \phi\right.\right. \\
& \left.\left.-\partial_{\beta \beta}^{2} \mu\right](\mathbf{r}, 0)\right\rangle,
\end{aligned}
$$

and its symmetrized form

$$
\begin{aligned}
\mathscr{R}(\mathbf{r}, \mathbf{r}, t)+\mathscr{R}(\mathbf{r}, \mathbf{r},-t) \\
=\frac{1}{D}\left\langle\left[\left(\partial_{t}+v_{\alpha} \partial_{\alpha}\right) \phi\right](\mathbf{r}, t)\left[\left(\partial_{t}+v_{\beta} \partial_{\beta}\right) \phi\right](\mathbf{r}, 0)\right\rangle \\
\quad-\frac{1}{2 D} \nabla_{(2)}^{2}\left\langle\left[\left(\partial_{t}+v_{\alpha} \partial_{\alpha}\right) \phi\right](\mathbf{r}, t) \mu(\mathbf{r}, 0)\right\rangle \\
\quad-\frac{1}{2 D} \nabla_{(2)}^{2}\left\langle\left[\left(\partial_{t}+v_{\alpha} \partial_{\alpha}\right) \phi\right](\mathbf{r}, 0) \mu(\mathbf{r}, t)\right\rangle .
\end{aligned}
$$

We see that the first two lines of Eq. (F6) can be written

$$
\begin{aligned}
& \frac{1}{2}\left\langle\left[\left(\partial_{t}+v_{\alpha} \partial_{\alpha}\right) \phi\right](\mathbf{r}, t) \mu(\mathbf{r}, 0)\right\rangle+\frac{1}{2}\left\langle\left[\left(\partial_{t}+v_{\alpha} \partial_{\alpha}\right) \phi\right](\mathbf{r}, 0) \mu(\mathbf{r}, t)\right\rangle \\
& =\nabla_{(2)}^{-2} \mathscr{C}(\mathbf{r}, \mathbf{r}, t)-D \nabla_{(2)}^{-2}[\mathscr{R}(\mathbf{r}, \mathbf{r}, t)+\mathscr{R}(\mathbf{r}, \mathbf{r},-t)], \quad(\mathrm{F} 11)
\end{aligned}
$$

where we employed the definition (49) for the correlation.

To obtain the last line in the expression for the entropy production (F6), we now consider the response with respect to the perturbation $\boldsymbol{\Sigma}^{A} \rightarrow \boldsymbol{\Sigma}^{A}-\varepsilon$, with $\varepsilon_{\alpha \beta}$ symmetric in the exchange of $\alpha$ and $\beta$ as well as traceless. The dynamic action at linear order in $\varepsilon$ reads

$$
\begin{aligned}
\delta \mathcal{A}= & -\frac{1}{2 \eta D} \int_{\mathbf{r}, t} \partial_{\beta} \varepsilon_{\alpha \beta} \nabla^{-2}\left[\left(\partial_{t}+v_{\gamma} \partial_{\gamma}\right) v_{\alpha}\right. \\
& \left.-\eta \nabla^{2} v_{\alpha}-\partial_{\gamma} \Sigma_{\alpha \gamma}^{A}+\partial_{\alpha} p\right] \\
& -\frac{1}{4 \eta D} \int_{\mathbf{r}, t} \partial_{\alpha} \partial_{\gamma} \varepsilon_{\alpha \gamma} \nabla^{-4}\left(\partial_{\beta} v_{\mu} \partial_{\mu} v_{\beta}\right. \\
& \left.-\partial_{\beta} \partial_{\mu} \Sigma_{\beta \mu}^{A}+\nabla^{2} p\right)+\mathcal{O}\left(\varepsilon^{2}\right) .
\end{aligned}
$$

Now, employing a formula analogous to Eq. (32), the response follows as

$$
\begin{aligned}
\mathcal{R}(\mathbf{r}, \mathbf{r}, t)= & -\frac{1}{2 \eta D}\left\langlev _ { \alpha \beta } ( \mathbf { r } , t ) \partial _ { \beta } \nabla ^ { - 2 } \left[\left(\partial_{t}+v_{\gamma} \partial_{\gamma}\right) v_{\alpha}\right.\right. \\
& \left.\left.-\eta \nabla^{2} v_{\alpha}-\partial_{\gamma} \Sigma_{\alpha \gamma}^{A}+\partial_{\alpha} p\right](\mathbf{r}, 0)\right\rangle \\
& +\frac{1}{4 \eta D}\left\langlev _ { \alpha \beta } ( \mathbf { r } , t ) \partial _ { \alpha } \partial _ { \beta } \nabla ^ { - 4 } \left(\partial_{\gamma} v_{\mu} \partial_{\mu} v_{\gamma}\right.\right. \\
& \left.\left.-\partial_{\gamma} \partial_{\mu} \Sigma_{\gamma \mu}^{A}+\nabla^{2} p\right)(\mathbf{r}, 0)\right\rangle .
\end{aligned}
$$

We now observe that we only need the integral over space: 


$$
\begin{aligned}
\int \mathcal{R}(\mathbf{r}, \mathbf{r}, t) d \mathbf{r}= & \frac{1}{2 D} \int\left\langle v_{\alpha \beta}(\mathbf{r}, t) v_{\alpha \beta}(\mathbf{r}, 0)\right\rangle d \mathbf{r} \\
& +\frac{1}{4 \eta D} \int\left\langle v_{\alpha \beta}(\mathbf{r}, t) \boldsymbol{\Sigma}_{\alpha \beta}(\mathbf{r}, 0)\right\rangle d \mathbf{r} \\
& +\frac{1}{4 \eta D} \int\left\langle v_{\alpha}(\mathbf{r}, t)\left(\partial_{t}+v_{\gamma} \partial_{\gamma}\right) v_{\alpha}(\mathbf{r}, 0)\right\rangle d \mathbf{r}
\end{aligned}
$$

where we have used the incompressibility condition and integration by parts to eliminate both the pressure term and the contribution given by the third and the fourth line of Eq. (F13). In the $t \rightarrow 0$ limit, we have

$$
\begin{aligned}
\lim _{t \rightarrow 0} \int\left\langle v_{\alpha}(\mathbf{r}, t)\left(\partial_{t}+v_{\gamma} \partial_{\gamma}\right) v_{\alpha}(\mathbf{r}, 0)\right\rangle d \mathbf{r} \\
\quad=\int\left\langle\frac{1}{2} \partial_{t} v_{\alpha}^{2}(\mathbf{r}, 0)+v_{\alpha} v_{\gamma} \partial_{\gamma} v_{\alpha}(\mathbf{r}, 0)\right\rangle d \mathbf{r}=0 .
\end{aligned}
$$

Using the definition of $\mathscr{C}$ in Eq. (49), we obtain

$$
\begin{aligned}
\lim _{t \rightarrow 0} \int[\mathcal{R}(\mathbf{r}, \mathbf{r}, t)+\mathcal{R}(\mathbf{r}, \mathbf{r},-t)] d \mathbf{r} \\
=\frac{1}{D} \lim _{t \rightarrow 0} \int \mathscr{C}(\mathbf{r}, \mathbf{r}, t) d \mathbf{r} \\
\quad+\frac{1}{4 \eta D} \lim _{t \rightarrow 0} \int\left\langle v_{\alpha \beta}(\mathbf{r}, t) \boldsymbol{\Sigma}_{\alpha \beta}(\mathbf{r}, 0)\right\rangle d \mathbf{r} \\
\quad+\frac{1}{4 \eta D} \lim _{t \rightarrow 0} \int\left\langle v_{\alpha \beta}(\mathbf{r}, 0) \boldsymbol{\Sigma}_{\alpha \beta}(\mathbf{r}, t)\right\rangle d \mathbf{r} .
\end{aligned}
$$

Plugging Eqs. (F10) and (F16) into Eq. (F6), we finally get

$$
\begin{aligned}
\mathcal{S}_{\phi, \mathbf{v}}= & \frac{1}{D} \lim _{t \rightarrow 0} \int \nabla_{(2)}^{-2}\{D[\mathscr{R}(\mathbf{r}, \mathbf{r}, t)+\mathscr{R}(\mathbf{r}, \mathbf{r},-t)] \\
& -\mathscr{C}(\mathbf{r}, \mathbf{r}, t)\} d \mathbf{r} \\
& +\frac{2 \eta}{D} \lim _{t \rightarrow 0} \int\{\mathcal{C}(\mathbf{r}, \mathbf{r}, t)-D[\mathcal{R}(\mathbf{r}, \mathbf{r}, t)+\mathcal{R}(\mathbf{r}, \mathbf{r},-t)]\} d \mathbf{r} .
\end{aligned}
$$

The generalized Harada-Sasa relation (50) is deduced by Fourier transforming in space and time.

[1] M. C. Marchetti, J. F. Joanny, S. Ramaswamy, T. B. Liverpool, J. Prost, Madan Rao, and R. Aditi Simha, Hydrodynamics of Soft Active Matter, Rev. Mod. Phys. 85, 1143 (2013).

[2] T. Vicsek, A. Czirók, E. Ben-Jacob, I. Cohen, and O. Shochet, Novel Type of Phase Transition in a System of Self-Driven Particles, Phys. Rev. Lett. 75, 1226 (1995).

[3] G. Grégoire and H. Chaté, Onset of Collective and Cohesive Motion, Phys. Rev. Lett. 92, 025702 (2004).
[4] A. P. Solon, H. Chaté, and J. Tailleur, From Phase to Microphase Separation in Flocking Models: The Essential Role of Nonequilibrium Fluctuations, Phys. Rev. Lett. 114, 068101 (2015).

[5] J. Deseigne, O. Dauchot, and H. Chaté, Collective Motion of Vibrated Polar Disks, Phys. Rev. Lett. 105, 098001 (2010).

[6] A. Bricard, J.-B. Caussin, N. Desreumaux, O. Dauchot, and D. Bartolo, Emergence of Macroscopic Directed Motion in Populations of Motile Colloids, Nature (London) 503, 95 (2013).

[7] H. H. Wensink, J. Dunkel, S. Heidenreich, K. Drescher, R. E. Goldstein, H. Löwen, and J. M. Yeomans, Mesoscale Turbulence in Living Fluids, Proc. Natl. Acad. Sci. U.S.A. 109, 14308 (2012).

[8] V. Schaller, C. Weber, C. Semmrich, E. Frey, and A. R. Bausch, Polar Patterns of Driven Filaments, Nature (London) 467, 73 (2010).

[9] Y. Sumino, K. H. Nagai, Y. Shitaka, D. Tanaka, K. Yoshikawa, H. Chaté, and K. Oiwa, Large-Scale Vortex Lattice Emerging from Collectively Moving Microtubules, Nature (London) 483, 448 (2012).

[10] J. Tailleur and M.E. Cates, Statistical Mechanics of Interacting Run-and-Tumble Bacteria, Phys. Rev. Lett. 100, 218103 (2008).

[11] Y. Fily and M. Cristina Marchetti, Athermal Phase Separation of Self-Propelled Particles with No Alignment, Phys. Rev. Lett. 108, 235702 (2012).

[12] M. E. Cates and J. Tailleur, Motility-Induced Phase Separation, Annu. Rev. Condens. Matter Phys. 6, 219 (2015).

[13] S. C. Takatori, W. Yan, and J. F. Brady, Swim Pressure: Stress Generation in Active Matter, Phys. Rev. Lett. 113, 028103 (2014).

[14] X. Yang, M. Lisa Manning, and M. Cristina Marchetti, Aggregation and Segregation of Confined Active Particles, Soft Matter 10, 6477 (2014).

[15] T. Speck, J. Bialké, A. M. Menzel, and H. Löwen, Effective Cahn-Hilliard Equation for the Phase Separation of Active Brownian Particles, Phys. Rev. Lett. 112, 218304 (2014).

[16] A. P. Solon, M. E. Cates, and J. Tailleur, Active Brownian Particles and Run-and-Tumble Particles: A Comparative Study, Eur. Phys. J. Spec. Top. 224, 1231 (2015).

[17] F. Ginot, I. Theurkauff, D. Levis, C. Ybert, L. Bocquet, L. Berthier, and C. Cottin-Bizonne, Nonequilibrium Equation of State in Suspensions of Active Colloids, Phys. Rev. X 5, 011004 (2015).

[18] S. C. Takatori and J. F. Brady, Towards a Thermodynamics of Active Matter, Phys. Rev. E 91, 032117 (2015).

[19] T. F. F. Farage, P. Krinninger, and J. M. Brader, Effective Interactions in Active Brownian Suspensions, Phys. Rev. E 91, 042310 (2015).

[20] U. Marini Bettolo Marconi and C. Maggi, Towards a Statistical Mechanical Theory of Active Fluids, Soft Matter 11, 8768 (2015).

[21] A. P. Solon, J. Stenhammar, M. E. Cates, Y. Kafri, and J. Tailleur, Generalized Thermodynamics of Phase Equilibria in Scalar Active Matter, arXiv:1609.03483.

[22] L. F. Cugliandolo, The Effective Temperature, J. Phys. A 44, 483001 (2011). 
[23] D. Loi, S. Mossa, and L. F. Cugliandolo, Effective Temperature of Active Matter, Phys. Rev. E 77, 051111 (2008).

[24] D. Loi, S. Mossa, and L. F. Cugliandolo, Effective Temperature of Active Complex Matter, Soft Matter 7, 3726 (2011).

[25] D. Levis and L. Berthier, From Single-Particle to Collective Effective Temperatures in an Active Fluid of Self-Propelled Particles, Europhys. Lett. 111, 60006 (2015).

[26] B. Lander, J. Mehl, V. Blickle, C. Bechinger, and U. Seifert, Noninvasive Measurement of Dissipation in Colloidal Systems, Phys. Rev. E 86, 030401 (2012).

[27] D. Mizuno, C. Tardin, C. F. Schmidt, and F. C. MacKintosh, Nonequilibrium Mechanics of Active Cytoskeletal Networks, Science 315, 370 (2007).

[28] C. Wilhelm, Out-of-Equilibrium Microrheology Inside Living Cells, Phys. Rev. Lett. 101, 028101 (2008).

[29] F. Gallet, D. Arcizet, P. Bohec, and A. Richert, Power Spectrum of Out-of-Equilibrium Forces in Living Cells: Amplitude and Frequency Dependence, Soft Matter 5, 2947 (2009).

[30] É. Fodor, M. Guo, N. S. Gov, P. Visco, D. A. Weitz, and F. van Wijland, Activity-Driven Fluctuations in Living Cells, Europhys. Lett. 110, 48005 (2015).

[31] W. W. Ahmed, E. Fodor, M. Almonacid, M. Bussonnier, M.-H. Verlhac, N. S. Gov, P. Visco, F. van Wijland, and T. Betz, Active Mechanics Reveal Molecular-Scale Force Kinetics in Living Oocytes, arXiv:1510.08299.

[32] T. Bodineau and B. Derrida, Cumulants and Large Deviations of the Current through Non-equilibrium Steady States, C.R. Phys. 8, 540 (2007).

[33] T. Bodineau and B. Derrida, Current Fluctuations in Nonequilibrium Diffusive Systems: An Additivity Principle, Phys. Rev. Lett. 92, 180601 (2004).

[34] L. Bertini, A. De Sole, D. Gabrielli, G. Jona-Lasinio, and C. Landim, Current Fluctuations in Stochastic Lattice Gases, Phys. Rev. Lett. 94, 030601 (2005).

[35] L. Bertini, A. De Sole, D. Gabrielli, G. Jona-Lasinio, and C. Landim, Macroscopic Fluctuation Theory, Rev. Mod. Phys. 87, 593 (2015).

[36] S. Pilgram, A. N. Jordan, E. V. Sukhorukov, and M. Büttiker, Stochastic Path Integral Formulation of Full Counting Statistics, Phys. Rev. Lett. 90, 206801 (2003).

[37] C. Battle, C. P. Broedersz, N. Fakhri, V. F. Geyer, J. Howard, C.F. Schmidt, and F.C. MacKintosh, Broken Detailed Balance at Mesoscopic Scales in Active Biological Systems, Science 352, 604 (2016).

[38] U. Seifert, Stochastic Thermodynamics, Fluctuation Theorems and Molecular Machines, Rep. Prog. Phys. 75, 126001 (2012).

[39] J. L. Lebowitz and H. Spohn, A Gallavotti-Cohen-Type Symmetry in the Large Deviation Functional for Stochastic Dynamics, J. Stat. Phys. 95, 333 (1999).

[40] C. Maes, The Fluctuation Theorem as a Gibbs Property, J. Stat. Phys. 95, 367 (1999).

[41] J. Kurchan, Fluctuation Theorem for Stochastic Dynamics, J. Phys. A 31, 3719 (1998).

[42] D. A. Egolf, Equilibrium Regained: From Nonequilibrium Chaos to Statistical Mechanics, Science 287, 101 (2000).
[43] S.-W. Wang, K. Kawaguchi, S.-i. Sasa, and L.-H. Tang, Entropy Production of Nano Systems with Timescale Separation, Phys. Rev. Lett. 117, 070601 (2016).

[44] S. Bo and A. Celani, Entropy Production in Stochastic Systems with Fast and Slow Time-Scales, J. Stat. Phys. 154, 1325 (2014).

[45] D. A. Egolf, Equilibrium Regained: From Nonequilibrium Chaos to Statistical Mechanics, Science 287, 101 (2000).

[46] L. Cerino and A. Puglisi, Entropy Production for Velocity-Dependent Macroscopic Forces: The Problem of Dissipation without Fluctuations, Europhys. Lett. 111, 40012 (2015).

[47] T. Harada and S.-i. Sasa, Equality Connecting Energy Dissipation with a Violation of the Fluctuation-Response Relation, Phys. Rev. Lett. 95, 130602 (2005).

[48] P.-S. Shim, H.-M. Chun, and J. D. Noh, Macroscopic Time-Reversal Symmetry Breaking at a Nonequilibrium Phase Transition, Phys. Rev. E 93, 012113 (2016).

[49] C. Kwon, J. Yeo, H. K. Lee, and H. Park, Anomalous Entropy Production in the Presence of MomentumDependent Forces, arXiv:1506.02339 [Phys. Rev. Lett. (to be published)].

[50] D. Chaudhuri, Active Brownian Particles: Entropy Production and Fluctuation Response, Phys. Rev. E 90, 022131 (2014).

[51] D. Chaudhuri, Entropy Production by Active Particles: Coupling of Odd and Even Functions of Velocity, Phys. Rev. E 94, 032603 (2016).

[52] É. Fodor, C. Nardini, M. E. Cates, J. Tailleur, P. Visco, and F.van Wijland, How Far from Equilibrium Is Active Matter?, Phys. Rev. Lett. 117, 038103 (2016).

[53] R. Wittkowski, A. Tiribocchi, J. Stenhammar, R. J. Allen, D. Marenduzzo, and M. E. Cates, Scalar $\varphi 4$ Field Theory for Active-Particle Phase Separation, Nat. Commun. 5, 4351 (2014).

[54] J. Stenhammar, A. Tiribocchi, R. J. Allen, D. Marenduzzo, and M. E. Cates, Continuum Theory of Phase Separation Kinetics for Active Brownian Particles, Phys. Rev. Lett. 111, 145702 (2013).

[55] M. E. Cates and J. Tailleur, When Are Active Brownian Particles and Run-and-Tumble Particles Equivalent? Consequences for Motility-Induced Phase Separation, Europhys. Lett. 101, 20010 (2013).

[56] P. C. Hohenberg and B. I. Halperin, Theory of Dynamic Critical Phenomena, Rev. Mod. Phys. 49, 435 (1977).

[57] P. M. Chaikin and T. C. Lubensky, Principles of Condensed Matter Physics (Cambridge University Press, Cambridge, England, 2000), Vol. 1.

[58] M. Kardar, G. Parisi, and Y.-C. Zhang, Dynamic Scaling of Growing Interfaces, Phys. Rev. Lett. 56, 889 (1986).

[59] S. F. Edwards and D. R. Wilkinson, The Surface Statistics of a Granular Aggregate, in Proceedings of the Royal Society of London A: Mathematical, Physical and Engineering Sciences (The Royal Society, London, 1982), Vol. 381, pp. 17-31.

[60] W. G. Faris and G. Jona-Lasinio, Large Fluctuations for a Nonlinear Heat Equation with Noise, J. Phys. A 15, 3025 (1982). 
[61] H. Touchette, The Large Deviation Approach to Statistical Mechanics, Phys. Rep. 478, 1 (2009).

[62] H. Touchette and R. J. Harris, Large Deviation Approach to Nonequilibrium Systems, edited by R. Klages, W. Just, and C. Jarzynski, Nonequilibrium Statistical Physics of Small Systems: Fluctuation Relations and Beyond (WileyVCH, Weinheim, 2013), p. 335.

[63] J. Tailleur, J. Kurchan, and V. Lecomte, Mapping Out-ofEquilibrium into Equilibrium in One-Dimensional Transport Models, J. Phys. A 41, 505001 (2008).

[64] F. Bouchet, K. Gawedzki, and C. Nardini, Perturbative Calculation of Quasi-potential in Non-equilibrium Diffusions: A Mean-Field Example, J. Stat. Phys. 163, 1157 (2016).

[65] B. Derrida, Microscopic versus Macroscopic Approaches to Non-equilibrium Systems, J. Stat. Mech. (2011) $\mathrm{P} 01030$.

[66] D. S. Dean, Langevin Equation for the Density of a System of Interacting Langevin Processes, J. Phys. A 29, L613 (1996).

[67] M. Doi, Second Quantization Representation for Classical Many-Particle System, J. Phys. A 9, 1465 (1976).

[68] L. Peliti, Path Integral Approach to Birth-Death Processes on a Lattice, J. Phys. 46, 1469 (1985).

[69] H.-K. Janssen, On a Lagrangean for Classical Field Dynamics and Renormalization Group Calculations of Dynamical Critical Properties, Z. Phys. B 23, 377 (1976).

[70] J. Tailleur, J. Kurchan, and V. Lecomte, Mapping Nonequilibrium onto Equilibrium: The Macroscopic Fluctuations of Simple Transport Models, Phys. Rev. Lett. 99, 150602 (2007).

[71] K. Mallick, M. Moshe, and H. Orland, A Field-Theoretic Approach to Non-equilibrium Work Identities, J. Phys. A 44, 095002 (2011).

[72] T. Leonard, B. Lander, U. Seifert, and T. Speck, Stochastic Thermodynamics of Fluctuating Density Fields: Nonequilibrium Free Energy Differences under CoarseGraining, J. Chem. Phys. 139, 204109 (2013).

[73] P. I. Hurtado, C. Pérez-Espigares, J. J. del Pozo, and P. L. Garrido, Symmetries in Fluctuations Far from Equilibrium, Proc. Natl. Acad. Sci. U.S.A. 108, 7704 (2011).

[74] G. Gradenigo, A. Puglisi, and A. Sarracino, Entropy Production in Non-equilibrium Fluctuating Hydrodynamics, J. Chem. Phys. 137, 014509 (2012).

[75] S. Toyabe, T. Okamoto, T. Watanabe-Nakayama, H. Taketani, S. Kudo, and E. Muneyuki, Nonequilibrium Energetics of a Single F1-ATPase Molecule, Phys. Rev. Lett. 104, 198103 (2010).

[76] S. Toyabe, H.-R. Jiang, T. Nakamura, Y. Murayama, and M. Sano, Experimental Test of a New Equality: Measuring Heat Dissipation in an Optically Driven Colloidal System, Phys. Rev. E 75, 011122 (2007).

[77] É. Fodor, W. W. Ahmed, M. Almonacid, M. Bussonnier, N. S. Gov, M.-H. Verlhac, T. Betz, P. Visco, and F. van Wijland, Nonequilibrium Dissipation in Living Oocytes, Europhys. Lett. 116, 30008 (2016).

[78] M. Paoluzzi, C. Maggi, U. Marini Bettolo Marconi, and N. Gnan, Critical Phenomena in Active Matter, arXiv:1609.03483 [Phys. Rev. Lett. (to be published)].
[79] K. Sekimoto, Stochastic Energetics (Springer, Berlin, 2010), Vol. 799.

[80] L. Onsager and S. Machlup, Fluctuations and Irreversible Processes, Phys. Rev. 91, 1505 (1953).

[81] P. C. Martin, E. D. Siggia, and H. A. Rose, Statistical Dynamics of Classical Systems, Phys. Rev. A 8, 423 (1973).

[82] C. De Dominicis, A Lagrangian Version of HalperinHohenberg-Ma Models for the Dynamics of Critical Phenomena, Nuovo Cimento 12, 567 (1975).

[83] N. G. Van Kampen, Stochastic Processes in Physics and Chemistry (Elsevier, New York, 1992), Vol. 1.

[84] J. Stenhammar, R. Wittkowski, D. Marenduzzo, and M. E. Cates, Light-Induced Self-Assembly of Active Rectification Devices, Sci. Adv. 2, e1501850 (2016).

[85] R. Kubo, The Fluctuation-Dissipation Theorem, Rep. Prog. Phys. 29, 255 (1966).

[86] L. Berthier and J. Kurchan, Non-equilibrium Glass Transitions in Driven and Active Matter, Nat. Phys. 9, 310 (2013).

[87] J. M. Deutsch and O. Narayan, Energy Dissipation and Fluctuation Response for Particles in Fluids, Phys. Rev. E 74, 026112 (2006).

[88] T. Harada, Macroscopic Expression Connecting the Rate of Energy Dissipation with the Violation of the Fluctuation Response Relation, Phys. Rev. E 79, 030106R (2009).

[89] D. Villamaina, A. Puglisi, and A. Vulpiani, The Fluctuation Dissipation Relation in Sub-diffusive Systems: The Case of Granular Single-File Diffusion, J. Stat. Mech. (2008) L10001.

[90] A. Tiribocchi, R. Wittkowski, D. Marenduzzo, and M. E. Cates, Active Model H: Scalar Active Matter in a Momentum-Conserving Fluid, Phys. Rev. Lett. 115, 188302 (2015).

[91] A. J. Bray, Theory of Phase-Ordering Kinetics, Adv. Phys. 43, 357 (1994).

[92] E. Tjhung, C. Nardini, R. Wittkowski, and E. M. Cates, Light-Induced Self-Assembly of Active Rectification Devices (unpublished).

[93] E. Bertin, M. Droz, and G. Grégoire, Boltzmann and Hydrodynamic Description for Self-Propelled Particles, Phys. Rev. E 74, 022101 (2006).

[94] T. B. Liverpool and M. Cristina Marchetti, Bridging the Microscopic and the Hydrodynamic in Active Filament Solutions, Europhys. Lett. 69, 846 (2005).

[95] E. Bertin, H. Chaté, F. Ginelli, S. Mishra, A. Peshkov, and S. Ramaswamy, Mesoscopic Theory for Fluctuating Active Nematics, New J. Phys. 15, 085032 (2013).

[96] T. Ihle, Kinetic Theory of Flocking: Derivation of Hydrodynamic Equations, Phys. Rev. E 83, 030901 (2011).

[97] A. G. Thompson, J. Tailleur, M.E. Cates, and R. A. Blythe, Lattice Models of Nonequilibrium Bacterial Dynamics, J. Stat. Mech. (2011) P02029.

[98] M. E. Cates, D. Marenduzzo, I. Pagonabarraga, and J. Tailleur, Arrested Phase Separation in Reproducing Bacteria Creates a Generic Route to Pattern Formation, Proc. Natl. Acad. Sci. U.S.A. 107, 11715 (2010).

[99] L. Chen, J. Toner, and C. F. Lee, Critical Phenomenon of the Order-Disorder Transition in Incompressible Active Fluids, New J. Phys. 17, 042002 (2015). 
[100] S. Mishra, R. Aditi Simha, and S. Ramaswamy, A Dynamic Renormalization Group Study of Active Nematics, J. Stat. Mech. (2010) P02003.

[101] J. Toner and Y. Tu, Long-Range Order in a TwoDimensional Dynamical XY Model: How Birds Fly Together, Phys. Rev. Lett. 75, 4326 (1995).

[102] L. Chen and J. Toner, Universality for Moving Stripes: A Hydrodynamic Theory of Polar Active Smectics, Phys. Rev. Lett. 111, 088701 (2013).

[103] C. W. Gardiner et al., Handbook of Stochastic Methods (Springer, Berlin, 1985), Vol. 3.

[104] A. W. C. Lau and T. C. Lubensky, State-Dependent Diffusion: Thermodynamic Consistency and Its Path Integral Formulation, Phys. Rev. E 76, 011123 (2007).
[105] C. Aron, D. G. Barci, L. F. Cugliandolo, Z. Gonzalez Arenas, and G.S. Lozano, Dynamical Symmetries of Markov Processes with Multiplicative White Noise, J. Stat. Mech. (2016) 053207.

[106] C. Aron, G. Biroli, and L. F. Cugliandolo, Symmetries of Generating Functionals of Langevin Processes with Colored Multiplicative Noise, J. Stat. Mech. (2010) P11018.

[107] H. K. Janssen, Lecture Notes in Physics in Dynamical Critical Phenomena and Related Topics (1979).

[108] P. E. Kloeden, E. Platen, and H. Schurz, Numerical Solution of SDE through Computer Experiments (Springer Science \& Business Media, New York, 2012). 